\title{
The Degrees of Freedom of Compute-and-Forward
}

\author{
Urs Niesen, and Phil Whiting
}

\begin{abstract}
We analyze the asymptotic behavior of compute-and-forward relay networks in the regime of high signal-tonoise ratios. We consider a section of such a network consisting of $K$ transmitters and $K$ relays. The aim of the relays is to reliably decode an invertible function of the messages sent by the transmitters. An upper bound on the capacity of this system can be obtained by allowing full cooperation among the transmitters and among the relays, transforming the network into a $K \times K$ multiple-input multiple-output (MIMO) channel. The number of degrees of freedom of compute-and-forward is hence at most $K$. In this paper, we analyze the degrees of freedom achieved by the lattice coding implementation of compute-and-forward proposed recently by Nazer and Gastpar. We show that this lattice implementation achieves at most $2 /(1+1 / K) \leq 2$ degrees of freedom, thus exhibiting a very different asymptotic behavior than the MIMO upper bound. This raises the question if this gap of the lattice implementation to the MIMO upper bound is inherent to compute-and-forward in general. We answer this question in the negative by proposing a novel compute-and-forward implementation achieving $K$ degrees of freedom.
\end{abstract}

\section{INTRODUCTION}

The two central problems of reliable communication over a wireless relay network are the signal interactions introduced by the wireless medium and the additive noise experienced at the nodes in the network. Traditional approaches of dealing with these problems fall broadly into two categories. On the one hand, intermediate relays in the network can try to completely remove the receiver noise. The decodeand-forward scheme (see [1]-[3], among others) falls into this category. While this solves the problem of noisy reception, its performance is adversely affected by the signal interactions, which are usually avoided by careful scheduling of transmissions. On the other hand, intermediate relays can try to make use of the signal interactions introduced by the channel either by not removing the additive noise at all, or by only removing it partially. Schemes such as amplify-and-forward (see, e.g., [2], [4]-[6]) or compress-andforward (see, e.g., [1], [3], [7]-[10]) fall into this category. Since noise is not or only partially removed at the relays, these schemes suffer from noise accumulation.

A third approach, referred to as either compute-and-forward [11], [12], physical-layer network coding [13], [14], or analog network coding [15], aims to both harness the signal interactions introduced by the channel and remove the noise at the relays. This is achieved by allowing the relays to decode noiseless functions of the transmitted messages. At the destination node all the information streams are combined to determine the original messages being sent. In this paper, we examine the design and performance of such schemes.

A small example illustrates the approach, see Fig. 1. Consider a section of a larger relay network with 2 transmitters and 2 relays. The channel gains $\left(h_{m, k}\right)$ between the transmitters and the relays are assumed to be constant and known throughout the network. The transmitters have access independent messages $w_{1}, w_{2}$, which are separately encoded, modulated, and then sent over the channel. The relays receive a linear combination of these transmitted signals corrupted by additive noise. Each relay decodes independently; however, the receivers do not aim to decode the original messages $w_{1}, w_{2}$. Rather, each relay $m$ decodes an intermediate quantity $u_{m}$, which is a noiseless function of the messages $w_{1}$ and $w_{2}$. Crucially, these functions are chosen to be adapted to the channel gains. In other words, the computation of the functions $u_{1}, u_{2}$ is aided by the signal interactions introduced by the channel. Following the decoding

U. Niesen and P. Whiting are with the Mathematics of Networks and Communications Research Department, Bell Labs, Alcatel-Lucent. Emails: urs.niesen@alcatel-lucent.com, pwhiting@research.bell-labs.com.

The material in this paper was presented in part at the 2011 IEEE International Symposium on Information Theory.

This work was supported in part by AFOSR under grant FA9550-09-1-0317. 
stage, the decoded functions $u_{1}, u_{2}$ are combined and inverted to recover the original messages $w_{1}, w_{2}$. This combining and inverting of the decoded functions is to be interpreted as taking place at the destination node (not explicitly modeled in this scenario), which is interested only in the original messages.

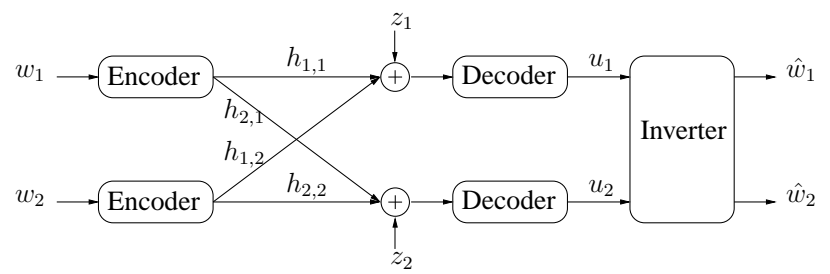

Fig. 1. A section of a relay network with two transmitters and two relays.

In [11], Nazer and Gastpar propose an ingenious coding scheme for compute-and-forward using lattice codes (see [16], [17], among others) at each transmitter. These lattice codes have the property that any integer linear combination of two codewords is again a codeword. Due to the additive nature of the channel, each relay receives a linear combination of the lattice codewords (which is again a codeword) plus some additive noise. The relays then decode the linear combination of the codewords, removing the noise. The relays thus decode a noiseless function of the messages. In terms of our example with two sources and relays, we see that the decoded quantities $u_{1}, u_{2}$ are linear functions of the messages $w_{1}, w_{2}$ in this case. Assuming the resulting system of linear equations is invertible, the original messages $w_{1}$ and $w_{2}$ can be recovered at the final destination from $u_{1}$ and $u_{2}$.

However, there is a subtle difficulty with this approach that we have neglected in the above description. The lattice property of the codes ensures only that any integer linear combination of codewords is again a codeword, whereas the linear combination computed by the wireless channel can have arbitrary real (or complex) coefficients. To overcome this difficulty, [11] proposes to scale the received channel output so that the scaled received linear combination of codewords is close to an integer linear combination. In general, the larger the scaling factor the better the approximation, increasing achievable rates. At the same time, a larger scaling factor results in amplification of noise, decreasing achievable rates.

We hence see that there is a tradeoff between closeness of approximation and noise amplification. This tradeoff is a central theme in the field of Diophantine approximation, which studies the approximation of real numbers by rationals, and we will refer to this as the Diophantine tradeoff in compute-and-forward. The rates achievable by the lattice coding implementation of compute-and-forward in [11] are not given by an analytic expression, but rather as the solution to an optimization problem, in which this tradeoff appears implicitly. It is hence not clear how significant the loss due to this Diophantine tradeoff is.

In this paper, we show that the loss in rate due to this tradeoff is indeed significant at high but still realistic values of signal-to-noise ratio (SNR), say $20 \mathrm{~dB}$ and above. In particular, for the two-user example discussed earlier, we show that due to this Diophantine tradeoff the compute-and-forward scheme in [11] achieves only one degree of freedom (capacity pre-log factor), the same as time sharing between the transmitters. In other words, in the two-user case, the compute-and-forward implementation in [11] and time sharing have the same high-SNR behavior. For the general case with $K$ transmitters and $K$ relays, we show that the lattice scheme achieves at most $2 /(1+1 / K) \leq 2$ degrees of freedom. While potentially better than time sharing, this is considerably worse than the MIMO upper bound of $K$ degrees of freedom that would be achievable with full cooperation among the transmitters and among the relays.

This negative result raises the question as to whether this Diophantine tradeoff and the associated loss are inherent to compute-and-forward as a scheme in general or whether they are an artifact of the implementation in [11]. We show that the latter is the case and that compute-and-forward in general does not suffer from this tradeoff. To this end, we propose a novel implementation of compute-and-forward that achieves $K$ degrees of freedom, matching the MIMO upper bound. Thus, compute-and-forward can achieve the same asymptotic rates as if cooperation among the transmitters and among the relays were allowed. 
The proposed achievable scheme introduces the concept of signal alignment, related to the alignment of interference. This alignment of signals is crucial to achieve the $K$ degrees of freedom upper bound, and indicates that the compute-and-forward problem and the interference channel problem are closely related.

The remainder of this paper is organized as follows. Section $\Pi$ provides a general formulation of the compute-and-forward setting. Section III states the main results. Proofs are presented in Sections IV VI] Section VII contains concluding remarks.

\section{Problem Statement And Notation}

\section{A. Notational Conventions}

Throughout this paper, we use the following notational conventions. Vectors and matrices are written in bold font in lower and upper case, respectively, e.g., $\boldsymbol{h}$ and $\boldsymbol{H}$. For a matrix $\boldsymbol{H}$, its transpose is denoted by $\boldsymbol{H}^{\top}$, and its determinant by $\operatorname{det}(\boldsymbol{H})$. For a vector $\boldsymbol{h}$, we write $\|\boldsymbol{h}\|$ for its Euclidean norm. We denote Lebesgue measure by $\mu$. We say that a property holds for almost every $\boldsymbol{H}$ if the set $B$ of $\boldsymbol{H}$ for which the property does not hold has Lebesgue measure $\mu(B)$ equal to zero. Finally, all logarithms are to the base 2 , and therefore channel capacities are expressed in bits per channel use.

\section{B. Problem Statement}

We consider a section of a relay network with $K$ transmitters and $K$ relays modeled by a discrete-time real Gaussian channel 11 The channel output $y_{m}[t]$ at receiver $m \in\{1, \ldots, K\}$ and time $t \in \mathbb{N}$ is

$$
y_{m}[t] \triangleq \sum_{k=1}^{K} h_{m, k} x_{k}[t]+z_{m}[t] .
$$

Here $x_{k}[t] \in \mathbb{R}$ is the channel input at transmitter $k \in\{1, \ldots, K\}, h_{m, k} \in \mathbb{R}$ is the channel gain between transmitter $k$ and receiver $m$, and $z_{m}[t] \in \mathbb{R}$ is additive white Gaussian noise with zero mean and unit variance. Note that the channel gains $\left(h_{m, k}\right)$ are deterministic and constant across time. As such, they are known throughout the network. To simplify notation, let the row vector

$$
\boldsymbol{h}_{m} \triangleq\left(\begin{array}{llll}
h_{m, 1} & h_{m, 2} & \cdots & h_{m, K}
\end{array}\right)
$$

be the channel gains to receiver $m$, and set

$$
\boldsymbol{H} \triangleq\left(\begin{array}{c}
\boldsymbol{h}_{1} \\
\boldsymbol{h}_{2} \\
\vdots \\
\boldsymbol{h}_{K}
\end{array}\right)
$$

Transmitter $k$ has access to an independent message $w_{k}$ uniformly distributed over $\left\{0,1, \ldots, W_{k}-1\right\}$. The goal of receiver $m$ is to compute the (deterministic) function

$$
u_{m} \triangleq a_{m}\left(w_{1}, w_{2}, \ldots, w_{K}\right) \in\left\{0,1, \ldots, U_{m}-1\right\} .
$$

Since $a_{m}$ is a deterministic function, its range can contain at most $U_{m} \leq \prod_{k=1}^{K} W_{k}$ elements. We impose that the messages $\left(w_{k}\right)$ can be recovered from the decoded equations $\left(u_{m}\right)$, i.e., that the vector map induced by the $K$ functions $\left(a_{m}\right)$ is invertible.

Formally, a block code of length $T$ and power constraint $P$ consists of $K$ encoders

$$
f_{k}:\left\{0, \ldots, W_{k}-1\right\} \rightarrow \mathbb{R}^{T}
$$

\footnotetext{
${ }^{1}$ Throughout this paper, we assume real channels. Using arguments similar to the ones in [18], [19], the results can be extended to hold for complex channels as well.
} 
for $k \in\{1, \ldots, K\}$, mapping the message $w_{k}$ to channel inputs

$$
\left(x_{k}[t]\right)_{t=1}^{T} \triangleq f_{k}\left(w_{k}\right)
$$

such that

$$
\frac{1}{T}\left\|f_{k}\left(w_{k}\right)\right\|^{2} \leq P
$$

and $K$ decoders

$$
\phi_{m}: \mathbb{R}^{T} \rightarrow\left\{0,1, \ldots, U_{m}-1\right\}
$$

for $m \in\{1, \ldots, K\}$, mapping the channel outputs $\left(y_{m}[t]\right)_{t=1}^{T}$ to the estimate

$$
\hat{u}_{m} \triangleq \phi_{m}\left(\left(y_{m}[t]\right)_{t=1}^{T}\right)
$$

of $u_{m}$. The probability of error of this block code is

$$
\mathbb{P}\left(\cup_{m \in\{1, \ldots, K\}}\left\{\hat{u}_{m} \neq u_{m}\right\}\right) .
$$

Observe that the probability of error is defined with respect to the equations $u_{m}$ and not the original messages $w_{k}$. The (sum) rate of this block code is

$$
\frac{1}{T} \sum_{k=1}^{K} \log \left(W_{k}\right) .
$$

A rate $R\left(\boldsymbol{H}, P,\left(a_{m}\right)\right)$ is achievable if for every $\eta>0$ there exists a block code of length $T$ and power constraint $P$ with probability of error less than $\eta$ and rate at least $R\left(\boldsymbol{H}, P,\left(a_{m}\right)\right)$. The computation capacity for functions $\left(a_{m}\right)$, denoted by $C\left(\boldsymbol{H}, P,\left(a_{m}\right)\right)$, is defined as the supremum of achievable rates. Finally, define the computation capacity

$$
C(\boldsymbol{H}, P) \triangleq \sup _{\left(a_{m}\right)} C\left(\boldsymbol{H}, P,\left(a_{m}\right)\right)
$$

where the supremum is over all invertible (deterministic) functions $\left(a_{m}\right)$.

Note that in this definition of computation capacity, it is irrelevant which functions the receivers decode, as long as all the decoded equations allow recovery of the original messages. This requirement is best understood in the context of a larger relay network, in which the channel considered here is only one component of the network, and the receivers here correspond to intermediate relays. The invertibility of the map $\left(a_{m}\right)$ guarantees that collectively the decoded equations $\left(u_{m}\right)$ at these relays contain all the information about the messages $\left(w_{k}\right)$ at the transmitters. However, the decoded equations have to be deterministic, i.e., all noise introduced by the channel has to be removed at the relays. This ensures that noise is not forwarded further down the larger relay network. These two requirements (invertibility and noise removal) are the essence of the compute-and-forward approach. We point out that decode-and-forward is a special case of the above definition in which the function $a_{m}$ are given by

$$
u_{m}=a_{m}\left(w_{1}, w_{2}, \ldots, w_{K}\right)=w_{m}
$$

for all $m$. On the other hand, schemes like amplify-and-forward or compress-and-forward do not satisfy the above definition, since they compute randomized (i.e., noisy) functions of the messages.

While the above definition of computation capacity allows for arbitrary functions $a_{m}$ it is worth mentioning the special case of linear functions. In this case, receiver $m$ aims to compute the function

$$
u_{m} \triangleq \sum_{k=1}^{K} a_{m, k} w_{k}
$$


with $a_{m, k} \in \mathbb{R} 2$ Define the row vector

$$
\boldsymbol{a}_{m} \triangleq\left(\begin{array}{llll}
a_{m, 1} & a_{m, 2} & \cdots & a_{m, K}
\end{array}\right)
$$

and the corresponding matrix

$$
\boldsymbol{A} \triangleq\left(\begin{array}{c}
a_{1} \\
a_{2} \\
\vdots \\
a_{K}
\end{array}\right)
$$

The messages $\left(w_{k}\right)$ can in this case be recovered from the decoded equations $\left(u_{m}\right)$ if the matrix $\boldsymbol{A}$ is full rank. With slight abuse of notation, we write $C(\boldsymbol{H}, P, \boldsymbol{A})$ for the computation capacity for the linear function determined by the coefficient matrix $\boldsymbol{A}$.

In the remainder of his paper, we will be interested in the degrees of freedom of the computation capacity $C(\boldsymbol{H}, P)$ defined as

$$
\lim _{P \rightarrow \infty} \frac{C(\boldsymbol{H}, P)}{\frac{1}{2} \log (P)}
$$

assuming the limit exists. If this limit is equal to $D$, then

$$
C(\boldsymbol{H}, P)=\frac{D}{2} \log (P)+o(\log (P))
$$

as $P \rightarrow \infty$. Thus, the degrees of freedom describe the behavior of $C(\boldsymbol{H}, P)$ at high SNR. Since the $o(\log (P))$ approximation alone can be quite weak, we will provide tighter second-order asymptotics as well.

\section{MAin Results}

Nazer and Gastpar [11, Theorems 1 and 2] provide an achievable scheme based on lattice codes for computation of linear equations over the channel (1), showing that, for $\boldsymbol{A} \in \mathbb{Z}^{K \times K}$,

$$
\begin{aligned}
C(\boldsymbol{H}, P, \boldsymbol{A}) & \geq R_{\mathrm{L}}(\boldsymbol{H}, P, \boldsymbol{A}) \\
& \triangleq \sum_{k=1}^{K} \min _{m: a_{m, k} \neq 0} R_{\mathrm{L}}\left(\boldsymbol{h}_{m}, P, \boldsymbol{a}_{m}\right) \\
& \triangleq \sum_{k=1}^{K} \min _{m: a_{m, k} \neq 0}\left(\frac{1}{2} \log \left(1+P\left\|\boldsymbol{h}_{m}\right\|^{2}\right)-\frac{1}{2} \log \left(\left\|\boldsymbol{a}_{m}\right\|^{2}+P\left(\left\|\boldsymbol{h}_{m}\right\|^{2}\left\|\boldsymbol{a}_{m}\right\|^{2}-\left(\boldsymbol{h}_{m} \boldsymbol{a}_{m}^{\top}\right)^{2}\right)\right)\right)
\end{aligned}
$$

is achievable. We emphasize that (2) is only valid for integer matrices $\boldsymbol{A} \in \mathbb{Z}^{K \times K}$. This restriction turns out to be a significant limitation, as we will see later.

Let us interpret the terms in the definition of $R_{\mathrm{L}}\left(\boldsymbol{h}_{m}, P, \boldsymbol{a}_{m}\right)$. The first term corresponds to the sum capacity of a multiple-access channel with channel gains $\boldsymbol{h}_{m}$. The second term represents the rate loss incurred by using the coefficients $\boldsymbol{a}_{m}$. This rate loss, governed by

$$
\left\|\boldsymbol{a}_{m}\right\|^{2}+P\left(\left\|\boldsymbol{h}_{m}\right\|^{2}\left\|\boldsymbol{a}_{m}\right\|^{2}-\left(\boldsymbol{h}_{m} \boldsymbol{a}_{m}^{\top}\right)^{2}\right)
$$

consists of two parts: the squared norm of $\boldsymbol{a}_{m}$, and the power $P$ times the gap arising from the CauchySchwarz inequality, which is therefore nonnegative. This second term is zero if and only if $\boldsymbol{a}_{m}$ and $\boldsymbol{h}_{m}$

\footnotetext{
${ }^{2}$ This setting can be slightly generalized by considering a vector of messages $\boldsymbol{w}_{k}$ (instead of a scalar $w_{k}$ ) and computing $\boldsymbol{u}_{k}$ by applying the same linear function to every component of $\boldsymbol{w}_{k}$. The distinction between the scalar and vector cases is immaterial for the purpose of this paper, and we will refer to both as linear computation.
} 
are collinear. Recall that $\boldsymbol{a}_{m}$ has integer components and can therefore not be chosen to be collinear to $\boldsymbol{h}_{m}$ in general. Denote by

$$
R_{\mathrm{L}}(\boldsymbol{H}, P) \triangleq \max _{\boldsymbol{A} \in \mathbb{Z}^{K \times K}: \operatorname{rank}(\boldsymbol{A})=K} R_{\mathrm{L}}(\boldsymbol{H}, P, \boldsymbol{A})
$$

the largest rate achievable with the lattice scheme proposed in [11] $]^{3}$

As mentioned earlier, the scheme by Nazer and Gastpar uses lattice codes, which have the property that every integer linear combination of two codewords is again a codeword. With this approach, the receivers directly decode the linear combinations $\left(u_{m}\right)$ and never explicitly decode the messages $\left(w_{k}\right)$. A different approach would be to choose $\boldsymbol{A}=\boldsymbol{I}$ so that $u_{m}=w_{m}$ for all $m$. This can be implemented by time sharing between all the transmitters, achieving a sum rate of at least

$$
\begin{aligned}
C(\boldsymbol{H}, P) & \geq C(\boldsymbol{H}, P, \boldsymbol{I}) \\
& \geq \sum_{k=1}^{K} \frac{1}{2 K} \log \left(1+K P\left|h_{k, k}\right|^{2}\right) .
\end{aligned}
$$

For $\boldsymbol{A}=\boldsymbol{I}$, the problem actually reduces to the standard interference channel, for which interference alignment achieves

$$
\begin{aligned}
C(\boldsymbol{H}, P) & \geq C(\boldsymbol{H}, P, \boldsymbol{I}) \\
& \geq \frac{K}{4} \log (P)-o(\log (P))
\end{aligned}
$$

as $P \rightarrow \infty$ for almost every channel matrix $\boldsymbol{H}[20]$. As $P \rightarrow \infty$, this rate is the best achievable for $\boldsymbol{A}=\boldsymbol{I}$ and almost every $\boldsymbol{H}$, as it is shown in [21] that

$$
C(\boldsymbol{H}, P, \boldsymbol{I}) \leq \frac{K}{4} \log (P)+o(\log (P)) .
$$

Finally, by allowing cooperation among the transmitters and among the receivers, the computation rate can be upper bounded by the capacity of the MIMO channel with the same channel matrix $\boldsymbol{H}$. This can be further upper bounded by relaxing the per-antenna power constraint to a sum power constraint, so that, by [22],

$$
C(\boldsymbol{H}, P) \leq \max \frac{1}{2} \log \operatorname{det}\left(\boldsymbol{I}+\boldsymbol{H} \boldsymbol{Q} \boldsymbol{H}^{\top}\right)
$$

where the maximization is over all covariance matrices $Q$ with trace at most $K P$.

To compare the upper bound (7) to the lower bounds (2), (4), and (5), it is insightful to consider their asymptotic behavior as power $P$ grows. The time-sharing lower bound (4) yields

$$
\liminf _{P \rightarrow \infty} \frac{C(\boldsymbol{H}, P)}{\frac{1}{2} \log (P)} \geq 1
$$

i.e., time sharing achieves one degree of freedom. The interference-alignment lower bound (5) yields

$$
\liminf _{P \rightarrow \infty} \frac{C(\boldsymbol{H}, P)}{\frac{1}{2} \log (P)} \geq K / 2
$$

for almost every channel matrix $\boldsymbol{H}$, i.e., interference alignment achieves $K / 2$ degrees of freedom. On the other hand, almost every channel matrix $\boldsymbol{H}$ has full rank, in which case the MIMO upper bound (7) yields

$$
\limsup _{P \rightarrow \infty} \frac{C(\boldsymbol{H}, P)}{\frac{1}{2} \log (P)} \leq K,
$$

${ }^{3}$ By [11, Lemma 1], a maximizing $\boldsymbol{A} \in \mathbb{Z}^{K \times K}$ exists. 
i.e., the corresponding MIMO channel has $K$ degrees of freedom. Thus, at high SNRs, time sharing and interference alignment behave very differently from the MIMO upper bound for almost every $\boldsymbol{H}$. Observe that by (6) any scheme using decode-and-forward, i.e., with coefficient matrix $\boldsymbol{A}=\boldsymbol{I}$, achieves at most $K / 2$ degrees of freedom for almost every $\boldsymbol{H}$. Hence, if we are to attain the upper bound of $K$ on the degrees of freedom, the use of general compute-and-forward (as opposed to simple decode-and-forward) will be necessary.

The behavior of the rate $R_{\mathrm{L}}$ achieved by the lattice scheme is more difficult to evaluate. If $\boldsymbol{H} \in \mathbb{Z}^{K \times K}$ has integer components and is invertible, we can set $\boldsymbol{A}=\boldsymbol{H}$ in (2) to obtain

$$
R_{\mathrm{L}}\left(\boldsymbol{h}_{m}, P, \boldsymbol{h}_{m}\right)=\frac{1}{2} \log \left(1+P\left\|\boldsymbol{h}_{m}\right\|^{2}\right)-\frac{1}{2} \log \left(\left\|\boldsymbol{h}_{m}\right\|^{2}\right) .
$$

Hence, in this case,

$$
\liminf _{P \rightarrow \infty} \frac{R_{\mathrm{L}}(\boldsymbol{H}, P)}{\frac{1}{2} \log (P)} \geq K .
$$

More generally, if $\boldsymbol{H} \in \mathbb{Q}^{K \times K}$ has rational components and is invertible, then there exists a $q \in \mathbb{N}$ such that $q \boldsymbol{H} \in \mathbb{Z}^{K \times K}$. Setting $\boldsymbol{A}=q \boldsymbol{H}$ in (2) yields that lattice coding achieves a rate of

$$
R_{\mathrm{L}}\left(\boldsymbol{h}_{m}, P, q \boldsymbol{h}_{m}\right)=\frac{1}{2} \log \left(1+P\left\|\boldsymbol{h}_{m}\right\|^{2}\right)-\frac{1}{2} \log \left(q^{2}\left\|\boldsymbol{h}_{m}\right\|^{2}\right),
$$

and again

$$
\liminf _{P \rightarrow \infty} \frac{R_{\mathrm{L}}(\boldsymbol{H}, P)}{\frac{1}{2} \log (P)} \geq K .
$$

Since $R_{\mathrm{L}} \leq C$, we obtain together with the MIMO upper bound (7) that for invertible $\boldsymbol{H} \in \mathbb{Q}^{K \times K}$

$$
\lim _{P \rightarrow \infty} \frac{R_{\mathrm{L}}(\boldsymbol{H}, P)}{\frac{1}{2} \log (P)}=K .
$$

In other words, for invertible $\boldsymbol{H}$ with rational components, the scheme based on lattice coding is asymptotically optimal. In particular, this implies that the lattice scheme significantly outperforms the schemes based on time sharing and based on interference alignment.

However, the requirement of rational channel gains $\boldsymbol{H}$ is quite strong. In fact, this event has Lebesgue measure zero. The question arises whether the behavior of the rate $R_{\mathrm{L}}$ achieved by the lattice scheme of [11] is significantly altered if we relax this assumption of rational channel gains. The next theorem shows that this is indeed the case. In fact, for almost all channel gains, the lattice scheme has an asymptotic behavior that is not significantly better than time sharing.

Theorem 1. For any $K \geq 2$ and almost every $\boldsymbol{H} \in \mathbb{R}^{K \times K}$ there exists a positive constant $c_{1}=c_{1}(K, \boldsymbol{H})$ such that for all $P \geq 3$

$$
R_{\mathrm{L}}(\boldsymbol{H}, P) \leq \frac{1}{1+1 / K} \log (P)+c_{1} \log \log (P) .
$$

In particular, this implies that for any $K \geq 2$ and almost every $\boldsymbol{H} \in \mathbb{R}^{K \times K}$

$$
\limsup _{P \rightarrow \infty} \frac{R_{\mathrm{L}}(\boldsymbol{H}, P)}{\frac{1}{2} \log (P)} \leq \frac{2}{1+1 / K} .
$$

We remark that, for $K=2$, Theorem 1 can be sharpened to

$$
\limsup _{P \rightarrow \infty} \max _{\boldsymbol{a}_{m} \in \mathbb{Z}^{2} \backslash\{\mathbf{0}\}} \frac{R_{\mathrm{L}}\left(\boldsymbol{h}_{m}, P, \boldsymbol{a}_{m}\right)}{\frac{1}{2} \log (P)} \leq 1 / 2,
$$

for almost every $\boldsymbol{h}_{m} \in \mathbb{R}^{2}$, so that

$$
\limsup _{P \rightarrow \infty} \frac{R_{\mathrm{L}}(\boldsymbol{H}, P)}{\frac{1}{2} \log (P)} \leq 1
$$


for almost every $\boldsymbol{H} \in \mathbb{R}^{2 \times 2}$.

Theorem 1 shows that for almost every channel matrix $\boldsymbol{H}$ there is only limited asymptotic gain over time sharing by using the lattice scheme in [11]. In particular, for $K=2$, time sharing and lattice coding achieve the same degrees of freedom. For large $K$, the upper bound in Theorem 1 is approximately $2-$ better than time sharing, but still far off from the $K / 2$ degrees of freedoms achievable with interference alignment and the MIMO upper bound of $K$ degrees of freedom. In other words, it seems to suggest that, at high SNR, compute-and-forward offers only limited advantage over standard coding schemes. This conclusion turns out to be misleading, as we will see later.

The bad asymptotic performance of the lattice scheme is due to the rate loss term (3). As pointed out earlier, to make the second term in (3) small, the coefficients $\boldsymbol{a}_{m}$ should be as close to collinear to the channel gains $\boldsymbol{h}_{m}$ as possible. However, since $\boldsymbol{a}_{m}$ is forced to be an integer vector, and since $\boldsymbol{h}_{m}$ is a real vector, this is in general only possible by increasing the norm of $\boldsymbol{a}_{m}$. This, in turn, increases the first term in (3). The tradeoff between the two terms in (3) is a main theme in the field of Diophantine approximation. In particular, the proof of Theorem 1 builds on a result of Khinchin to show that, for almost every channel gain $\boldsymbol{h}_{m}$, the coefficient vector $\boldsymbol{a}_{m}$ can only be close to collinear to $\boldsymbol{h}_{m}$ if $\left\|\boldsymbol{a}_{m}\right\|$ is large.

Example 1. Consider the channel vector $\boldsymbol{h}=\left(\begin{array}{ll}1 & h_{2}\end{array}\right)$ to one of the receiver. Consider

$$
\max _{\boldsymbol{a} \in \mathbb{Z}^{2} \backslash\{\mathbf{0}\}} R_{\mathrm{L}}(\boldsymbol{h}, P, \boldsymbol{a}),
$$

the maximal rate at which any (nontrivial) integer linear equation can be decoded at the receiver. From (9), we know that

$$
\limsup _{P \rightarrow \infty} \max _{\boldsymbol{a} \in \mathbb{Z}^{2} \backslash\{\mathbf{0}\}} \frac{R_{\mathrm{L}}(\boldsymbol{h}, P, \boldsymbol{a})}{\frac{1}{2} \log (P)} \leq 1 / 2
$$

for almost every $h_{2} \in \mathbb{R}$. On the other hand, for $h_{2} \in \mathbb{Q}$,

$$
\lim _{P \rightarrow \infty} \max _{\boldsymbol{a} \in \mathbb{Z}^{2} \backslash\{\mathbf{0}\}} \frac{R_{\mathrm{L}}(\boldsymbol{h}, P, \boldsymbol{a})}{\frac{1}{2} \log (P)}=1 .
$$

While these statements are only valid asymptotically as $P \rightarrow \infty$, this qualitative behavior is already visible at moderate values of SNR, as is depicted in Fig. 2.

We now introduce a different implementation of the compute-and-forward approach that achieves $K$ degrees of freedom, matching the asymptotic behavior of the MIMO upper bound (8). In other words, even though both the receivers and the transmitters are distributed, the proposed communication scheme achieves the same number of degrees of freedom as a centralized communication scheme in which all transmitters can cooperate and all receivers can cooperate.

Theorem 2. For every $K \geq 2$ and almost every $\boldsymbol{H} \in \mathbb{R}^{K \times K}$ there exist positive constants $c_{2}=c_{2}(K, \boldsymbol{H})$ and $c_{3}=c_{3}(K, \boldsymbol{H})$ such that for all $P \geq 2$

$$
\frac{K}{2} \log (P)-c_{2} \log \frac{K^{2}}{1+K^{2}}(P) \leq C(\boldsymbol{H}, P) \leq \frac{K}{2} \log (P)+c_{3} .
$$

In particular, this implies that for any $K \geq 2$ and almost every $\boldsymbol{H} \in \mathbb{R}^{K \times K}$,

$$
\lim _{P \rightarrow \infty} \frac{C(\boldsymbol{H}, P)}{\frac{1}{2} \log (P)}=K .
$$

Recall that the implementation of compute-and-forward in [11] uses lattice/linear codes together with output scaling. The aim of this output scaling is to make the scaled channel gains close to integer. The

\footnotetext{
${ }^{4}$ While 9 is stated for almost every $\boldsymbol{h} \in \mathbb{R}^{2}$, the same arguments can be used to show that 9) also holds for almost every $\boldsymbol{h}$ of the form (1 $h_{2}$ ).
} 

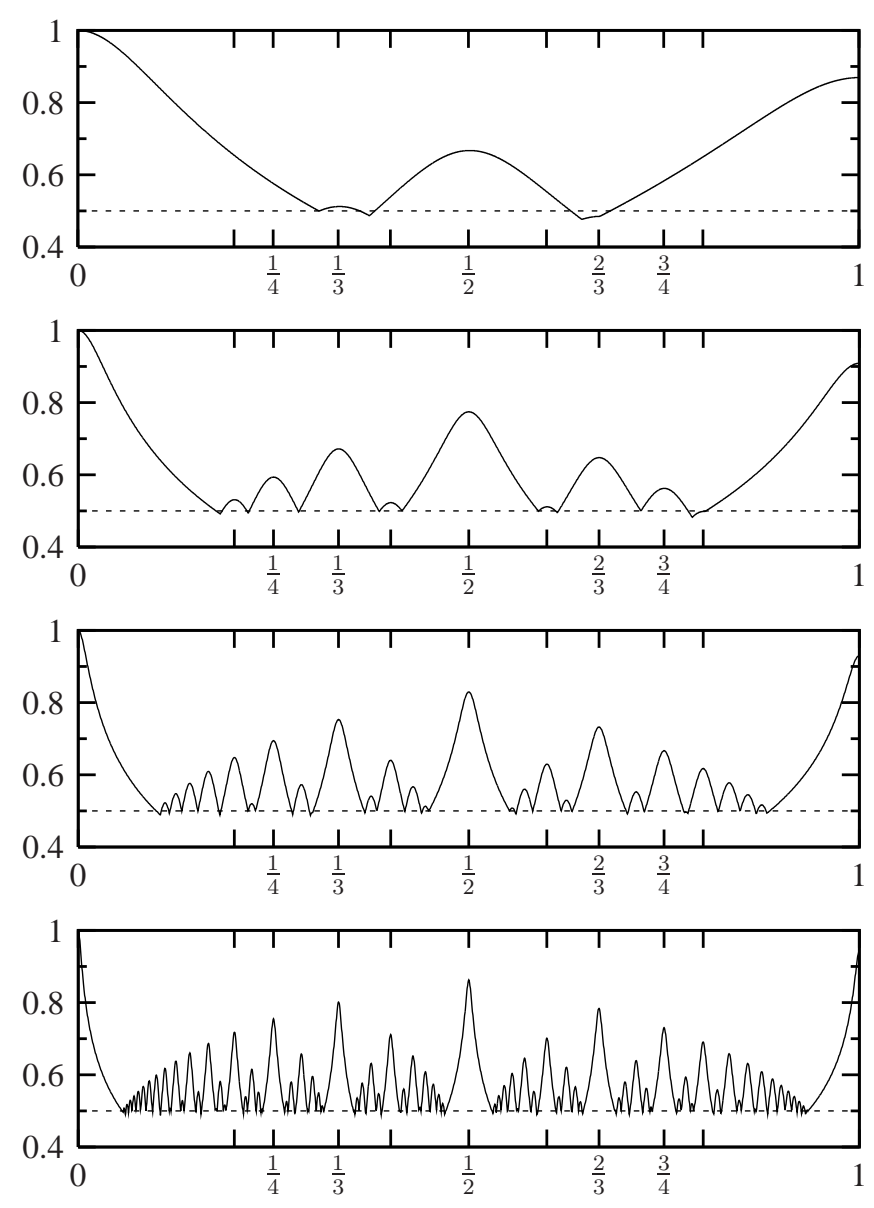

Fig. 2. Normalized rate $\max _{\boldsymbol{a}} R_{\mathrm{L}}(\boldsymbol{h}, P, \boldsymbol{a}) / \frac{1}{2} \log \left(1+\boldsymbol{h}^{2} P\right)$ achievable with lattice codes [11] with optimized coefficient vector $\boldsymbol{a} \in \mathbb{Z}^{2} \backslash\{\mathbf{0}\}$ for channel gain $\boldsymbol{h}=\left(\begin{array}{ll}1 & h_{2}\end{array}\right)$ as a function of $h_{2} \in[0,1]$. The plots are for a value $P$ of $20 \mathrm{~dB}, 30 \mathrm{~dB}, 40 \mathrm{~dB}$, and $50 \mathrm{~dB}$ (from top to bottom). As $P \rightarrow \infty$, the normalized rate converges to at most $1 / 2$ for almost every value of $h_{2}$. On the other hand, for $h_{2} \in \mathbb{Q}$ (a set of measure zero), the normalized rate converges to 1 . This limiting behavior can already be observed at the values of SNR shown here.

difficulty with this approach is that the scaling of the channel outputs amplifies the additive receiver noise. In order for the scaled channel gains to be close to integer, the scaling factor should be large. On the other hand, in order to have small noise amplification, the scaling factor should be small. These two conflicting requirements result in the Diophantine tradeoff mentioned in the introduction. This tradeoff can be observed in the tension between the two terms in (3) as discussed earlier.

Our proposed achievable scheme in Theorem 2 also uses linear codes at the transmitters. However, it avoids the scaling of the channel outputs and thereby the Diophantine tradeoff. Instead, we use a modulation scheme based on signal alignment over the real numbers to convert the real linear combinations produced by the channel into integer linear combinations. This step builds on a construction suggested recently for the alignment of interference in [20], [23], which itself is based on prior work on Diophantine approximation on manifolds [24], [25]. The proposed approach is best illustrated with an example.

Example 2. Consider again the $K=2$ case. Assume the channel gains are of the form

$$
\boldsymbol{H} \triangleq\left(\begin{array}{cc}
1 & h_{2} \\
h_{1} & 1
\end{array}\right) .
$$

Set the channel input to be

$$
\begin{aligned}
& x_{1} \triangleq \bar{w}_{1}, \\
& x_{2} \triangleq \bar{w}_{2},
\end{aligned}
$$


where both $\bar{w}_{k}$ are codewords from the same lattice code. The channel output is then

$$
\begin{aligned}
& y_{1}=\bar{w}_{1}+h_{2} \bar{w}_{2}+z_{1}, \\
& x_{2}=h_{1} \bar{w}_{1}+\bar{w}_{2}+z_{2} .
\end{aligned}
$$

Given that both codewords are from the same lattice code, one might hope that an integer combination of them might be decodable at higher rates than the individual messages themselves. However, the arguments in Theorem 1 show that, for almost all $\boldsymbol{H}$ and at high enough SNR, each receiver can essentially decode both $\bar{w}_{1}$ and $\bar{w}_{2}$ whenever it can decode an integer combination of them. This limits the computation rate to one degree of freedom.

A simple improvement over this scheme is to set

$$
\begin{aligned}
& x_{1} \triangleq \bar{w}_{1}, \\
& x_{2} \triangleq h_{1} \bar{w}_{2},
\end{aligned}
$$

The channel output is now

$$
\begin{aligned}
& y_{1}=\bar{w}_{1}+h_{1} h_{2} \bar{w}_{2}+z_{1}, \\
& x_{2}=h_{1}\left(\bar{w}_{1}+\bar{w}_{2}\right)+z_{2} .
\end{aligned}
$$

This results in the signals $\bar{w}_{1}$ and $\bar{w}_{2}$ to be both observed with the same effective channel gain $h_{1}$ at receiver two. In other words, we have signal alignment at the second receiver. However, the signals at the first receiver are still unaligned. This limits the computation rate to again only one degree of freedom.

To achieve alignment at both receivers, split the messages into two parts, and set

$$
\begin{aligned}
& x_{1} \triangleq \bar{w}_{1,1}+h_{1} h_{2} \bar{w}_{1,2}, \\
& x_{2} \triangleq h_{1} \bar{w}_{2,1}+h_{1}^{2} h_{2} \bar{w}_{2,2} .
\end{aligned}
$$

This results in the channel outputs

$$
\begin{aligned}
& y_{1}=\bar{w}_{1,1}+h_{1} h_{2}\left(\bar{w}_{1,2}+\bar{w}_{2,1}\right)+h_{1}^{2} h_{2}^{2} \bar{w}_{2,2}+z_{1}, \\
& x_{2}=h_{1}\left(\bar{w}_{1,1}+\bar{w}_{2,1}\right)+h_{1}^{2} h_{2}\left(\bar{w}_{1,2}+\bar{w}_{2,2}\right)+z_{2} .
\end{aligned}
$$

We now have partial alignment at both receivers. Receiver one decodes $\bar{w}_{1,1}, \bar{w}_{1,2}+\bar{w}_{2,1}$, and $\bar{w}_{2,2}$. Receiver two decodes $\bar{w}_{1,1}+\bar{w}_{2,1}$ and $\bar{w}_{1,2}+\bar{w}_{2,2}$. It can be shown that this achieves a computation rate of $4 / 3$ degrees of freedom.

By breaking the messages into more submessages and aligning them pairwise in the same manner, this construction achieves a computation rate approaching two degrees of freedom, as promised in Theorem 2

Remark 1: In the channel model (1), the channel gains $\boldsymbol{H}$ are assumed to be constant and as such known everywhere. In practice, this channel state information (CSI) would have to be estimated and distributed throughout the network, resulting in signaling overhead.

Having access to CSI at both the receivers and the transmitters is critical for the operation of the compute-and-forward scheme proposed here (achieving $K$ degrees of freedom) as well as for the interference alignment scheme in [20] (achieving $K / 2$ degrees of freedom). In contrast, the lattice coding implementation of compute-and-forward in [11] (and shown here to achieve at most 2 degrees of freedom) requires only CSI at the receivers but not at the transmitters. Whether the lattice coding scheme in [11] achieves the optimal degrees of freedom if the use of transmitter CSI is excluded is an open question.

Remark 2: Throughout this paper, we have been concerned almost exclusively with degrees of freedom. The second-order asymptotics in Theorem 2 are quite poor, especially for larger values of $K$. Deriving tighter approximations valid for moderate values of SNR is an interesting direction for further investigation. 


\section{PReliminaries: Diophantine ApProximation}

In all of the proofs, we will be using facts from Diophantine approximation. Here we provide the necessary background as well as some extensions of well-known results.

Let $h$ be a real and $a, q$ be integers. How well can $h$ be approximated by the ratio $a / q$ ? Since the rationals $\mathbb{Q}$ are dense in the reals $\mathbb{R}$, this can be done to any arbitrary degree of accuracy. However, to get a good approximation, the denominator $q$ will, in general, have to be large. The question then becomes one of quantifying the tradeoff between the quality of approximation and the size of $q$. Formally, the problem is to analyze the behavior of

$$
\min _{a \in \mathbb{Z}}|h-a / q|
$$

as a function of $q \in \mathbb{N}$ for fixed $h \in \mathbb{R}$. A result due to Khinchin (see, e.g., [26, Theorem 1]) states that if $\psi$ is a nonnegative function such that

$$
\sum_{q=1}^{\infty} q \psi(q)
$$

converges, then for almost every $h \in \mathbb{R}$ there exists a positive constant $c=c(h)$ such that

$$
\min _{a \in \mathbb{Z}}|h-a / q| \geq c \psi(q)
$$

for all $q \in \mathbb{N}$. On the other hand, if (11) diverges, then for almost every $h \in \mathbb{R}$ and every positive constant $c$, there are infinitely many values of $q \in \mathbb{N}$ such that

$$
\min _{a \in \mathbb{Z}}|h-a / q| \leq c \psi(q)
$$

The convergent and divergent parts of Khinchin's theorem show that for almost every $h \in \mathbb{R}$ the approximation error $|h-a / q|$ can be made to decay at least as fast as $O\left(q^{-2+\delta}\right)$ but no faster than $\Omega\left(q^{-2-\delta}\right)$ for any $\delta>0$.

The next lemma provides a simple generalization of the convergent part of Khinchin's theorem to more than one dimension and to approximations with denominator $\sqrt{q}$.

Lemma 3. Let $\psi: \mathbb{N} \rightarrow \mathbb{R}_{+}$. If

$$
\sum_{q=1}^{\infty}(\sqrt{q} \psi(q))^{K}<\infty
$$

then for almost every $\boldsymbol{h} \in \mathbb{R}^{K}$ there is a positive constant $c=c(K, \boldsymbol{h})$ such that

$$
\max _{k \in\{1, \ldots, K\}} \min _{a_{k} \in \mathbb{Z}}\left|h_{k}-a_{k} / \sqrt{q}\right| \geq c \psi(q)
$$

for all $q \in \mathbb{N}$.

The lemma implies that, for almost every $\boldsymbol{h} \in \mathbb{R}^{K}$, the approximation error

$$
\max _{k} \min _{a_{k} \in \mathbb{Z}}\left|h_{k}-a_{k} / \sqrt{q}\right|
$$

can decay no faster than $\Omega\left(q^{-1 / 2-1 / K-\delta}\right)$ for any $\delta>0$.

Proof: Let $B_{q}$ be the vectors $\boldsymbol{h} \in[0,1)^{K}$ such that

$$
\max _{k} \min _{a_{k} \in \mathbb{Z}}\left|h_{k}-a_{k} / \sqrt{q}\right| \leq \psi(q) .
$$

Since $h_{k} \in[0,1)$, the integer $a_{k}$ can be restricted to the set $\{0, \ldots,\lceil\sqrt{q}\rceil\}$ for all $k$. Setting $\boldsymbol{a}=\left(a_{k}\right)$, we see that a vector $\boldsymbol{h}$ is in $B_{q}$ if and only if it is at a $\ell_{\infty}$ distance of at most $\psi(q)$ of such a vector $\boldsymbol{a} / \sqrt{q}$. 
Thus, each $\boldsymbol{a} \in\{0, \ldots,\lceil\sqrt{q}\rceil\}^{K}$ contributes at most a subset of volume $(2 \psi(q))^{K}$ to $B_{q}$. Since there are at most $(\sqrt{q}+2)^{K}$ such vectors $\boldsymbol{a}$, we have

$$
\mu\left(B_{q}\right) \leq(\sqrt{q}+2)^{K}(2 \psi(q))^{K} .
$$

By the convergence assumption, this implies that

$$
\sum_{q=1}^{\infty} \mu\left(B_{q}\right) \leq \sum_{q=1}^{\infty}(\sqrt{q}+2)^{K}(2 \psi(q))^{K}<\infty .
$$

Applying the Borel-Cantelli lemma (see, e.g., [27, Theorem 1.6.1]), this shows that

$$
\mu\left(B_{q} \text { i.o. }\right)=0,
$$

where "i.o." stands for "infinitely often" (as a function of $q$ ). Thus, almost every $\boldsymbol{h} \in[0,1)^{K}$ satisfies (12) only finitely many times. Since $\mathbb{R}^{K}$ is the countable union of integer cubes $\prod_{k=1}^{K}\left[b_{k}, b_{k}+1\right)$, the same holds also for almost all $\boldsymbol{h} \in \mathbb{R}^{K}$.

Fix a $\boldsymbol{h} \in \mathbb{R}^{K}$ for which (12) holds only finitely many times. Then there exists a finite number $Q(\boldsymbol{h})$ such that

$$
\max _{k} \min _{a_{k} \in \mathbb{Z}}\left|h_{k}-a_{k} / \sqrt{q}\right| \geq \psi(q)
$$

for all $q \geq Q(\boldsymbol{h})$. Set

$$
c=c(K, \boldsymbol{h}) \triangleq \min \left\{1, \min _{q \in\{1, \ldots, Q(h)\}} \frac{\max _{k} \min _{a_{k} \in \mathbb{Z}}\left|h_{k}-a_{k} / \sqrt{q}\right|}{\psi(q)}\right\},
$$

and observe that $c$ is positive. Then

$$
\max _{k} \min _{a_{k} \in \mathbb{Z}}\left|h_{k}-a_{k} / \sqrt{q}\right| \geq c \psi(q)
$$

for all $q \in \mathbb{N}$, concluding the proof of the lemma.

We will also need a generalization of the convergent part of Khinchin's theorem to manifolds in Euclidean space. We start with a small example to illustrate the setting. Consider again the question of rational approximation in (10). This can be generalized to several dimensions as follows. Fix $\boldsymbol{H} \in \mathbb{R}^{K \times K}$; what is the behavior of

$$
\min _{a \in \mathbb{Z}}\left|\sum_{k, m} q_{m, k} h_{m, k}-a\right|
$$

as a function of $q_{m, k} \in \mathbb{Z}$ ? The generalization of Khinchin's theorem to this setting is referred to as Groshev's theorem.

A further generalization, and the one that will be needed in this paper, is to allow for functions of $\boldsymbol{H}$. Let $\mathcal{G}$ be a collection of functions $g: \mathbb{R}^{K \times K} \rightarrow \mathbb{R}$. Fix $\boldsymbol{H} \in \mathbb{R}^{K \times K}$; what is the behavior of

$$
\min _{a \in \mathbb{Z}}\left|\sum_{g \in \mathcal{G}} q_{g} g(\boldsymbol{H})-a\right|
$$

as a function of $q_{g} \in \mathbb{Z}$ ? In particular, we will be interested in the collection of functions

$$
\mathcal{G}_{L} \triangleq\left\{\prod_{k=1}^{K} \prod_{m=1}^{K} h_{m, k}^{s_{m, k}}: S \in\{0, \ldots, L-1\}^{K \times K}\right\} .
$$

In words, $\mathcal{G}_{L}$ is the collection of all monomials in the channel gains $\boldsymbol{H}$ with exponents between 0 and $L-1$. In the following, we will usually fix a particular realization of $\boldsymbol{H}$ and treat the set $\mathcal{G}_{L}$ as a collection of $L^{K^{2}}$ points in $\mathbb{R}$.

Remark 3: It is straightforward to verify that, for almost every $\boldsymbol{H} \in \mathbb{R}^{K \times K}$, all $L^{K^{2}}$ monomials in $\mathcal{G}_{L}$ evaluate to distinct numbers. This implies that, for almost every $\boldsymbol{H} \in \mathbb{R}^{K \times K}$, we have $\left|\mathcal{G}_{L}\right|=L^{K^{2}}$. 
Furthermore, again for almost all $\boldsymbol{H}$, every $g \in \mathcal{G}_{L}$ can be uniquely factorized into powers of $h_{m, k}$. In other words, to each $g$ corresponds a unique set of powers $\boldsymbol{S}$ such that

$$
g=\prod_{m, k} h_{m, k}^{s_{m, k}} .
$$

We refer to this as the unique factorization property. Given that we are only interested in results that hold for almost every channel matrix $\boldsymbol{H}$, we may assume in the following that $\mathcal{G}_{L}$ has this unique factorization property.

The following lemma is a special case of a more general result from [24], [25] (see also [20]).

Lemma 4. Let $\psi: \mathbb{N} \rightarrow \mathbb{R}_{+}$be a monotonically decreasing function and $L \in \mathbb{N}, L \geq 2$. If

$$
\sum_{q=1}^{\infty} q^{\left|\mathcal{G}_{L}\right|-2} \psi(q)<\infty
$$

then for almost every $\boldsymbol{H} \in \mathbb{R}^{K \times K}$ there is a positive constant $c=c(K, \boldsymbol{H})$ such that

$$
\min _{a \in \mathbb{Z}}\left|\sum_{g \in \mathcal{G}_{L}, g \neq 1} q_{g} g-a\right| \geq c \psi\left(\max _{g \in \mathcal{G}_{L}, g \neq 1}\left|q_{g}\right|\right)
$$

for all $\left(q_{g}\right) \in \mathbb{Z}^{\left|\mathcal{G}_{L}\right|-1} \backslash\{0\}$.

Lemma 4 implies that, for almost every $\boldsymbol{H} \in \mathbb{R}^{K \times K}$, the approximation error

$$
\min _{a \in \mathbb{Z}}\left|\sum_{g \in \mathcal{G}_{L}, g \neq 1} q_{g} g-a\right|
$$

can decay no faster than

$$
\Omega\left(\left(\max _{g \in \mathcal{G}_{L}, g \neq 1}\left|q_{g}\right|\right)^{-\left|\mathcal{G}_{L}\right|+1-\delta}\right)
$$

for any $\delta>0$.

\section{Proof of Theorem 1}

We want to upper bound the largest rate

$$
R_{\mathrm{L}}(\boldsymbol{H}, P) \triangleq \max _{\boldsymbol{A} \in \mathbb{Z}^{K \times K}: \operatorname{rank}(\boldsymbol{A})=K} R_{\mathrm{L}}(\boldsymbol{H}, P, \boldsymbol{A})
$$

achievable with the lattice coding scheme of [11]. From the above definition, we see that the coefficient matrix $\boldsymbol{A}$ can be chosen as a function of $P$. In particular, to each $P$ corresponds an optimal $\boldsymbol{A}=\boldsymbol{A}(P)$ maximizing the right-hand side of (14) 5 We consider this $\boldsymbol{A}$ in the following so that

$$
R_{\mathrm{L}}(\boldsymbol{H}, P)=R_{\mathrm{L}}(\boldsymbol{H}, P, \boldsymbol{A}(P)) .
$$

Recall that

$$
R_{\mathrm{L}}(\boldsymbol{H}, P, \boldsymbol{A})=\sum_{k=1}^{K} \min _{m: a_{m, k} \neq 0} R_{\mathrm{L}}\left(\boldsymbol{h}_{m}, P, \boldsymbol{a}_{m}\right),
$$

and that $R_{\mathrm{L}}\left(\boldsymbol{h}_{m}, P, \boldsymbol{a}_{m}\right)$ consists of two terms, the desired term

$$
\frac{1}{2} \log \left(1+P\left\|\boldsymbol{h}_{m}\right\|^{2}\right)
$$

and the loss term

$$
-\frac{1}{2} \log \left(\left\|\boldsymbol{a}_{m}\right\|^{2}+P\left(\left\|\boldsymbol{h}_{m}\right\|^{2}\left\|\boldsymbol{a}_{m}\right\|^{2}-\left(\boldsymbol{h}_{m} \boldsymbol{a}_{m}^{\top}\right)^{2}\right)\right) .
$$

${ }^{5}$ It can be shown that such an optimal $\boldsymbol{A}$ exists, see [11, Lemma 1]. If more than one maximizer exists, we choose one of them. 
We start by upper bounding $R_{\mathrm{L}}\left(\boldsymbol{h}_{m}, P, \boldsymbol{a}_{m}\right)$ for a fixed value of $m \in\{1, \ldots, K\}$. Together with (16), this yields an upper bound on $R_{\mathrm{L}}(\boldsymbol{H}, P, \boldsymbol{A})$ and hence on $R_{\mathrm{L}}(\boldsymbol{H}, P)$.

We can rewrite the quantity inside the logarithm of the loss term in $R_{\mathrm{L}}\left(\boldsymbol{h}_{m}, P, \boldsymbol{a}_{m}\right)$ as

$$
\begin{aligned}
\left\|\boldsymbol{a}_{m}\right\|^{2}+P\left(\left\|\boldsymbol{h}_{m}\right\|^{2}\left\|\boldsymbol{a}_{m}\right\|^{2}-\left(\boldsymbol{h}_{m} \boldsymbol{a}_{m}^{\top}\right)^{2}\right) & =\left\|\boldsymbol{a}_{m}\right\|^{2}+P\left\|\boldsymbol{h}_{m}\right\|^{2}\left\|\boldsymbol{a}_{m}\right\|^{2}\left(1-\cos ^{2}\left(\angle\left(\boldsymbol{h}_{m}, \boldsymbol{a}_{m}\right)\right)\right) \\
& =\left\|\boldsymbol{a}_{m}\right\|^{2}+P\left\|\boldsymbol{h}_{m}\right\|^{2}\left\|\boldsymbol{a}_{m}\right\|^{2} \sin ^{2}\left(\angle\left(\boldsymbol{h}_{m}, \boldsymbol{a}_{m}\right)\right) .
\end{aligned}
$$

Now, for $x \in[-\pi / 2, \pi / 2]$,

$$
\sin ^{2}(x) \geq \frac{4}{\pi^{2}} x^{2}
$$

and, for $\angle\left(\boldsymbol{h}_{m}, \boldsymbol{a}_{m}\right)$ measured in $\in[-\pi, \pi]$,

$$
\left|\angle\left(\boldsymbol{h}_{m}, \boldsymbol{a}_{m}\right)\right| \geq\left\|\frac{\boldsymbol{h}_{m}}{\left\|\boldsymbol{h}_{m}\right\|}-\frac{\boldsymbol{a}_{m}}{\left\|\boldsymbol{a}_{m}\right\|}\right\|
$$

by lower bounding the distance along the great circle on the unit sphere by its chordal distance. Since $R_{\mathrm{L}}\left(\boldsymbol{h}_{m}, P, \boldsymbol{a}_{m}\right)$ is invariant to multiplication of $\boldsymbol{a}_{m}$ by -1 , we can assume that $\angle\left(\boldsymbol{h}_{m}, \boldsymbol{a}_{m}\right) \in[-\pi / 2, \pi / 2]$ so that

$$
\begin{aligned}
\left\|\boldsymbol{a}_{m}\right\|^{2}+P\left(\left\|\boldsymbol{h}_{m}\right\|^{2}\left\|\boldsymbol{a}_{m}\right\|^{2}-\left(\boldsymbol{h}_{m} \boldsymbol{a}_{m}^{\top}\right)^{2}\right) & \geq\left\|\boldsymbol{a}_{m}\right\|^{2}+\frac{4}{\pi^{2}} P\left\|\boldsymbol{h}_{m}\right\|^{2}\left\|\boldsymbol{a}_{m}\right\|^{2}\left\|\frac{\boldsymbol{h}_{m}}{\left\|\boldsymbol{h}_{m}\right\|}-\frac{\boldsymbol{a}_{m}}{\left\|\boldsymbol{a}_{m}\right\|}\right\|^{2} \\
& \geq\left\|\boldsymbol{a}_{m}\right\|^{2}+\frac{4}{\pi^{2}} P\left\|\boldsymbol{h}_{m}\right\|^{2}\left\|\boldsymbol{a}_{m}\right\|^{2} \max _{1 \leq k \leq K}\left|\frac{h_{m, k}}{\left\|\boldsymbol{h}_{m}\right\|}-\frac{a_{m, k}}{\left\|\boldsymbol{a}_{m}\right\|}\right|^{2} \\
& \geq\left\|\boldsymbol{a}_{m}\right\|^{2}+\frac{4}{\pi^{2}} P\left\|\boldsymbol{h}_{m}\right\|^{2}\left\|\boldsymbol{a}_{m}\right\|^{2} \max _{1 \leq k \leq K-1}\left|\frac{h_{m, k}}{\left\|\boldsymbol{h}_{m}\right\|}-\frac{a_{m, k}}{\left\|\boldsymbol{a}_{m}\right\|}\right|^{2} .
\end{aligned}
$$

As we will see shortly, the restriction of the maximum in the last inequality to $k \in\{1, \ldots, K-1\}$ is necessary to decouple the (implicit) optimization over $\boldsymbol{a}_{m}$ into the first $K-1$ individual components $a_{k}$ and the magnitude $\left\|\boldsymbol{a}_{m}\right\|^{2}$.

Define

$$
\begin{aligned}
\tilde{\boldsymbol{h}}_{m} & \triangleq \frac{1}{\left\|\boldsymbol{h}_{m}\right\|}\left(\begin{array}{lll}
h_{m, 1} & \cdots & h_{m, K-1}
\end{array}\right), \\
q_{m} & \triangleq\left\|\boldsymbol{a}_{m}\right\|^{2} \in \mathbb{N}, \\
\psi_{m}\left(q_{m}\right) & \triangleq \max _{k \in\{1, \ldots, K-1\}}\left|\tilde{h}_{m, k}-a_{m, k} / \sqrt{q_{m}}\right| .
\end{aligned}
$$

With this, we can rewrite (17) as

$$
\left\|\boldsymbol{a}_{m}\right\|^{2}+P\left(\left\|\boldsymbol{h}_{m}\right\|^{2}\left\|\boldsymbol{a}_{m}\right\|^{2}-\left(\boldsymbol{h}_{m} \boldsymbol{a}_{m}^{\top}\right)^{2}\right) \geq q_{m}+\frac{4}{\pi^{2}} P\left\|\boldsymbol{h}_{m}\right\|^{2} q_{m} \psi_{m}^{2}\left(q_{m}\right) .
$$

We want to minimize the rate loss (18). The first term in the right-hand side of (18) is increasing in $q_{m}$. As we shall see, the second term is decreasing in $q_{m}$. Hence there is a tradeoff between the two terms that determines the optimal value of $q_{m}$.

The behavior of the approximation error $\psi_{m}\left(q_{m}\right)$ can be bounded using the convergent part of Khinchin's theorem in $K-1$ dimensions. To this end, note that

$$
\begin{aligned}
\psi_{m}\left(q_{m}\right) & \geq \min _{\boldsymbol{a}_{m} \in \mathbb{Z}^{K-1}} \max _{k \in\{1, \ldots, K-1\}}\left|\tilde{h}_{m, k}-a_{m, k} / \sqrt{q_{m}}\right| \\
& =\max _{k \in\{1, \ldots, K-1\}} \min _{a_{m, k} \in \mathbb{Z}}\left|\tilde{h}_{m, k}-a_{m, k} / \sqrt{q_{m}}\right|,
\end{aligned}
$$


which is of the form analyzed in Lemma 3. Applying Lemma 3 in $K-1$ dimensions shows then that for any fixed $\delta>0$ and almost every $\tilde{\boldsymbol{h}}_{m}$ there exists $c=c\left(K, \tilde{\boldsymbol{h}}_{m}\right)=c(K, \boldsymbol{h})>0$ such that

$$
\psi_{m}\left(q_{m}\right)>c q_{m}^{-1 / 2-1 /(K-1)-\delta}
$$

for all $q_{m} \in \mathbb{N}$. Observe that this lower bound holds for any choice of $\boldsymbol{a}_{m}$; in particular, the constant $c$ is uniform in $\boldsymbol{a}_{m}$. We can then continue to lower bound the loss term in $R_{\mathrm{L}}\left(\boldsymbol{h}_{m}, P, \boldsymbol{a}_{m}\right)$ as

$$
\begin{aligned}
q_{m}+\frac{4}{\pi^{2}} P\left\|\boldsymbol{h}_{m}\right\|^{2} q_{m} \psi_{m}^{2}\left(q_{m}\right) & \geq q_{m}+\frac{4}{\pi^{2}} c P\left\|\boldsymbol{h}_{m}\right\|^{2} q_{m}^{-2 /(K-1)-2 \delta} \\
& \geq \max \left\{q_{m}, \frac{4}{\pi^{2}} c P\left\|\boldsymbol{h}_{m}\right\|^{2} q_{m}^{-2 /(K-1)-2 \delta}\right\} .
\end{aligned}
$$

This shows the tradeoff between the two cost terms. Recall that we are allowed to choose $\boldsymbol{A}=\boldsymbol{A}(P)$, and hence also $q_{m}$, as a function of power $P$. Asymptotically, the optimal choice of $q_{m}$ is

$$
q_{m}=q_{m}(P)=\Theta\left(P^{(1+2 /(K-1)+2 \delta)^{-1}}\right),
$$

and hence

$$
q_{m}+P\left\|\boldsymbol{h}_{m}\right\|^{2} \frac{4}{\pi^{2}} q_{m} \psi_{m}^{2}\left(q_{m}\right) \geq \Omega\left(P^{(1+2 /(K-1)+2 \delta)^{-1}}\right)
$$

as $P \rightarrow \infty$.

Combined with (18), this shows that, for almost every $\tilde{\boldsymbol{h}}_{m}$,

$$
R_{\mathrm{L}}\left(\boldsymbol{h}_{m}, P, \boldsymbol{a}_{m}(P)\right) \leq \frac{1}{2} \log \left(1+P\left\|\boldsymbol{h}_{m}\right\|^{2}\right)-\frac{1}{2} \log \left(\Omega\left(P^{(1+2 /(K-1)+2 \delta)^{-1}}\right)\right)
$$

as $P \rightarrow \infty$. This implies that

$$
\limsup _{P \rightarrow \infty} \frac{R_{\mathrm{L}}\left(\boldsymbol{h}, P, \boldsymbol{a}_{m}(P)\right)}{\frac{1}{2} \log (P)} \leq \frac{2+\tilde{\delta}}{K+1+\tilde{\delta}}
$$

where we have set

$$
\tilde{\delta} \triangleq 2(K-1) \delta>0 .
$$

We have argued that (21) holds for almost every $\tilde{\boldsymbol{h}}_{m} \in \mathbb{R}^{K-1}$. It is shown in Appendix @ that this implies that (21) also holds for almost every $\boldsymbol{h}_{m} \in \mathbb{R}^{K}$.

Up to this point, we have analyzed the rate for a single receiver $m$. Using the definition of $R_{\mathrm{L}}(\boldsymbol{H}, P, \boldsymbol{A})$ in (16), this yields the upper bound

$$
\begin{aligned}
R_{\mathrm{L}}(\boldsymbol{H}, P, \boldsymbol{A}(P)) & =\sum_{k=1}^{K} \min _{m: a_{m, k} \neq 0} R_{\mathrm{L}}\left(\boldsymbol{h}_{m}, P, \boldsymbol{a}_{m}(P)\right) \\
& \leq K \max _{m \in\{1, \ldots, K\}} R_{\mathrm{L}}\left(\boldsymbol{h}_{m}, P, \boldsymbol{a}_{m}(P)\right)
\end{aligned}
$$

on the sum rate. Together with (21) and using the union bound over $m \in\{1, \ldots, K\}$, this implies that

$$
\limsup _{P \rightarrow \infty} \frac{R_{\mathrm{L}}(\boldsymbol{H}, P, \boldsymbol{A}(P))}{\frac{1}{2} \log (P)} \leq \frac{2+\tilde{\delta}}{1+1 / K+\tilde{\delta} / K}
$$

for almost every $\boldsymbol{H}$. As we have assumed that $\boldsymbol{A}(P)$ is the optimal coefficient matrix for power $P$, this implies by (15) that

$$
\limsup _{P \rightarrow \infty} \frac{R_{\mathrm{L}}(\boldsymbol{H}, P)}{\frac{1}{2} \log (P)} \leq \frac{2+\tilde{\delta}}{1+1 / K+\tilde{\delta} / K}
$$


for almost every $\boldsymbol{H}$. Since $\tilde{\delta}>0$ is arbitrary, we may take the limit as $\tilde{\delta} \rightarrow 0$ to obtain

$$
\limsup _{P \rightarrow \infty} \frac{R_{\mathrm{L}}(\boldsymbol{H}, P)}{\frac{1}{2} \log (P)} \leq \frac{2}{1+1 / K}
$$

yielding the desired upper bound on the degrees of freedom of the lattice scheme.

We now derive an estimate of the speed of convergence. Observe that we can choose $\delta$ in (19) as

$$
\delta=\delta\left(q_{m}\right)=\frac{2 \log \log \left(1+q_{m}\right)}{(K-1) \log \left(q_{m}\right)}
$$

and still satisfy the convergence condition in Lemma 3 in $K-1$ dimensions. The lower bound (20) on the loss term in $R_{\mathrm{L}}\left(\boldsymbol{h}_{m}, P, \boldsymbol{a}_{m}\right)$ then becomes

$$
\begin{aligned}
q_{m}+\frac{4}{\pi^{2}} P\left\|\boldsymbol{h}_{m}\right\|^{2} q_{m} \psi_{m}^{2}\left(q_{m}\right) & \geq \max \left\{q_{m}, \frac{4}{\pi^{2}} c P\left\|\boldsymbol{h}_{m}\right\|^{2} q_{m}^{-\frac{2}{K-1}} \log ^{-\frac{4}{K-1}}\left(1+q_{m}\right)\right\} \\
& \geq \log \frac{4}{K-1}\left(1+q_{m}\right) \max \left\{q_{m}, \frac{4}{\pi^{2}} c P\left\|\boldsymbol{h}_{m}\right\|^{2} q_{m}^{-\frac{2}{K-1}}\right\} .
\end{aligned}
$$

By [11, Lemma 1], we can restrict the optimization over $\boldsymbol{A}$ to matrices satisfying

$$
q_{m}=\left\|\boldsymbol{a}_{m}\right\|^{2} \leq\left\|\boldsymbol{h}_{m}\right\|^{2} P
$$

so that

$$
q_{m}+\frac{4}{\pi^{2}} P\left\|\boldsymbol{h}_{m}\right\|^{2} q_{m} \psi_{m}^{2}\left(q_{m}\right) \geq \log ^{-\frac{4}{K-1}}\left(1+\left\|\boldsymbol{h}_{m}\right\|^{2} P\right) \max \left\{q_{m}, \frac{4}{\pi^{2}} c P\left\|\boldsymbol{h}_{m}\right\|^{2} q_{m}^{-\frac{2}{K-1}}\right\} .
$$

We can now solve for the optimal $q_{m}$. Proceeding as before, we obtain an upper bound on the computation rate with lattice coding of

$$
\begin{aligned}
R_{\mathrm{L}}(\boldsymbol{H}, P) & \leq \max _{m \in\{1, \ldots, K\}} K\left(\frac{1}{2} \log \left(1+P\left\|\boldsymbol{h}_{m}\right\|^{2}\right)-\frac{1}{2} \log \Omega\left(\log ^{-\frac{4}{K-1}}\left(1+\left\|\boldsymbol{h}_{m}\right\|^{2} P\right) P^{\left(1+\frac{2}{K-1}\right)^{-1}}\right)\right) \\
& \leq \frac{1}{1+1 / K} \log (P)+O(\log \log (P))
\end{aligned}
$$

as $P \rightarrow \infty$. This implies that for any $K \geq 2$ and almost every $\boldsymbol{H} \in \mathbb{R}^{K \times K}$ there exists a positive constant $c_{1}=c_{1}(K, \boldsymbol{H})$ such that for all $P \geq 3$

$$
R_{\mathrm{L}}(\boldsymbol{H}, P) \leq \frac{1}{1+1 / K} \log (P)+c_{1} \log \log (P),
$$

proving the theorem.

\section{Proof of TheOREM 2}

The upper bound in Theorem 2 follows immediately from (7). We focus here on the lower bound showing that

$$
\liminf _{P \rightarrow \infty} \frac{C(\boldsymbol{H}, P)}{\frac{1}{2} \log (P)} \geq K
$$

We start with a high-level description of the scheme achieving this performance in Section VI-A. The detailed analysis can be found in Section VI-B. 


\section{A. Description of Communication Scheme}

The proposed coding scheme consists of two components: a modulation scheme and an outer code (see Fig. 3). The encoder $f_{k}$ for the outer code at transmitter $k$ maps the message $w_{k}$ into the sequence of coded symbols $\left(\bar{w}_{k}[t]\right)_{t=1}^{T}$. The modulator $\bar{f}_{k}$ at transmitter $k$ maps each coded symbol $\bar{w}_{k}[t]$ into a channel symbol $x_{k}[t]$. Thus, while the outer code produces a block of coded symbols, the modulation scheme operates on a single coded symbol to produce a single channel symbol. The encoder in the definition of computation capacity is the concatenation of these two encoding operations. At receiver $m$, the demodulator $\bar{\phi}_{m}$ computes $\hat{\bar{u}}_{m}[t]$ from the channel output $y_{m}[t]$, and the decoder $\phi_{m}$ for the outer code maps the sequence $\left(\hat{\bar{u}}_{m}[t]\right)_{t=1}^{T}$ into an estimate $\hat{u}_{m}$ of the desired function $u_{m}$. Both $u_{m}$ and $\bar{u}_{m}$ are defined as a function of $\left(w_{k}\right)$ and $\left(\bar{w}_{k}\right)$, respectively. The decoder in the definition of computation capacity is the concatenation of these two decoding operations.

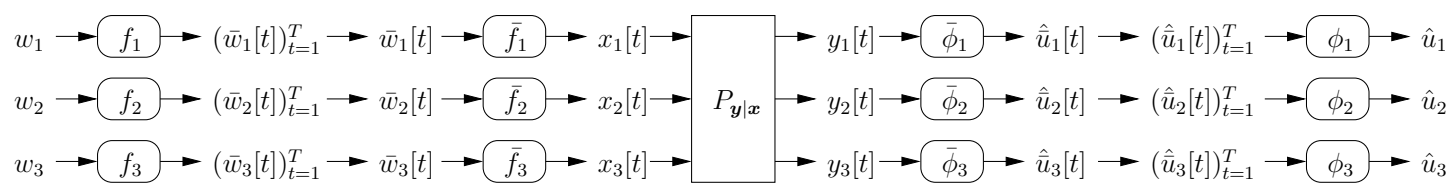

Fig. 3. Modulation scheme $\left(\left\{\bar{f}_{k}\right\},\left\{\bar{\phi}_{m}\right\}\right)$ together with outer code $\left(\left\{f_{k}\right\},\left\{\phi_{m}\right\}\right)$. At transmitter $k$, the message $w_{k}$ is mapped by the encoder $f_{k}$ of the outer code into the sequence $\left(\bar{w}_{k}[t]\right)_{t=1}^{T}$ of modulator inputs. Each $\bar{w}_{k}[t]$ is mapped by the modulator $\bar{f}_{k}$ to a channel input $x_{k}[t]$. At receiver $m$, the channel output $y_{m}[t]$ is mapped by the demodulator $\bar{\phi}_{m}$ into a demodulated equation $\hat{\bar{u}}_{m}[t]$. The sequence of these demodulated equations $\left(\hat{\bar{u}}_{m}[t]\right)_{t=1}^{T}$ is mapped by the decoder $\phi_{m}$ of the outer code to an estimate $\hat{u}_{m}$ of the desired equation $u_{m}$.

Note that in the description of the proposed achievable scheme we are using the following notational conventions. Quantities related to the outer code are denoted by standard font, i.e., $f_{k}, \phi_{m}, w_{k}, \ldots$ The corresponding quantities related to the modulation scheme are indicated by bars, i.e., $\bar{f}_{k}, \bar{\phi}_{m}, \bar{w}_{k}, \ldots$ Estimated quantities are indicated by hats, i.e., the output $\hat{u}_{m}$ of the decoder of the outer code is an estimate of the correct output $u_{m}$, and similarly the output $\hat{\bar{u}}_{m}$ of the demodulator is an estimate of the correct output $\bar{u}_{m}$.

The construction is as follows. Each message $w_{k}$ is split into $\left|\mathcal{G}_{L}\right|=L^{K^{2}}$ submessages $\left(w_{k, g}\right)_{g \in \mathcal{G}_{L}}$ with $\mathcal{G}_{L}$ defined in (13). Every $f_{k}$ encodes each of these submessages $w_{k, g}$ using the same linear code. Thus, all encoders $\left\{f_{k}\right\}$ are identical. The modulator $\bar{f}_{k}$ combines these $\left|\mathcal{G}_{L}\right|$ codewords into a single sequence of channel inputs.

Consider now receiver $m$. The channel to this receiver is in effect a $K$-user multiple-access channel (MAC). By splitting the transmitted message into submessage, we have transformed this $K$-user MAC into a $K\left|\mathcal{G}_{L}\right|$-user MAC, with each user corresponding to one submessage. The demodulator $\bar{\phi}_{m}$ splits this MAC at receiver $m$ into $\left|\mathcal{G}_{L+1}\right|$ subchannels. Through careful design of the modulators, this splitting can be done such that each of the resulting $\left|\mathcal{G}_{L+1}\right|$ MACs outputs a (noisy) sum of only $K$ out of the $K\left|\mathcal{G}_{L}\right|$ possible input signals. Observe that this channel is linear with integer channel coefficients. Hence, the linear codes used as outer code can now be efficiently decoded. The decoder $\phi_{m}$ of the outer code is thus chosen to recover the submessage corresponding to the sum of the $K$ codewords seen over this MAC. Decoding is shown to be possible with vanishing probability of error with a rate of order $\frac{1}{2\left|\mathcal{G}_{L+1}\right|} \log (P)$ for each of the submessages for large $P$. Moreover, it can be shown that the resulting collection of decoded functions is invertible.

Since there are $\left|\mathcal{G}_{L}\right|$ submessages for each of the $K$ transmitters, the sum rate achieved by this scheme is on the order of

$$
\frac{K\left|\mathcal{G}_{L}\right|}{2\left|\mathcal{G}_{L+1}\right|} \log (P)=\frac{K}{2(1+1 / L)^{K^{2}}} \log (P)
$$

The scheme achieves therefore

$$
\frac{K}{(1+1 / L)^{K^{2}}}
$$

degrees of freedom. For large $L$, this is approaches the $K$ degrees of freedom claimed in Theorem 2 


\section{B. Detailed Proof of Achievability}

The proof of the theorem consists of three steps. First, we show how the modulation scheme transforms the noisy linear combinations with real coefficients produced by the channel (1) into a system computing noisy linear combinations with integer coefficients. Second, we show how the outer code further transforms this modulated channel into a system computing noiseless linear combinations with integer coefficients. Third, we argue that the linear combinations produced by the outer code are invertible, i.e., the messages at the transmitters can be recovered from the computed linear combinations of all receivers.

We now describe the operations of the modulation scheme in detail (see Fig. 4). Recall the definition of $\mathcal{G}_{L}$ in (13) as the collection of all monomials in the channel gains with exponents between 0 and $L-1$. The input symbol $\bar{w}_{k}[t]$ to the modulator $\bar{f}_{k}$ at transmitter $k$ at time $t$ consists of $\left|\mathcal{G}_{L}\right|$ subsymbols

$$
\bar{w}_{k}[t] \triangleq\left(\bar{w}_{k, g}[t]\right)_{g \in \mathcal{G}_{L}}, \quad \bar{w}_{k, g}[t] \in\{0, \ldots, p-1\} \forall k, g, t
$$

for some $p, L \in \mathbb{N}$ to be chosen later. The output symbol $\hat{\bar{u}}_{m}[t]$ of the demodulator $\bar{\phi}_{m}$ at receiver $m$ at time $t$ consists of $\left|\mathcal{G}_{L+1}\right|$ subsymbols

$$
\hat{\bar{u}}_{m}[t] \triangleq\left(\hat{\bar{u}}_{m, g}[t]\right)_{g \in \mathcal{G}_{L+1}}, \quad \hat{\bar{u}}_{m, g}[t] \in\{0, \ldots, p-1\} \forall m, g, t .
$$

Note that the number of input and output subsymbols per time slot are not the same.

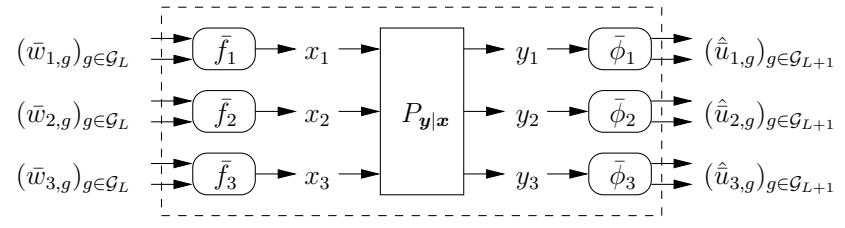

Fig. 4. Modulation scheme $\left(\left\{\bar{f}_{k}\right\},\left\{\bar{\phi}_{m}\right\}\right)$. The modulator $\bar{f}_{k}$ at transmitter $k$ takes $\left(\bar{w}_{k, g}\right)_{g \in \mathcal{G}_{L}}$ as input. The demodulator $\bar{\phi}_{m}$ at receiver $m$ produces $\left(\hat{\bar{u}}_{m, g}\right)_{g \in \mathcal{G}_{L+1}}$ as its output. Indicated by the dashed box is the modulated channel obtained by viewing the modulation scheme as part of the channel. This modulated channel is discrete and memoryless. All operations take place over a single time slot $t$; the dependence of $\bar{w}_{k, g}, x_{k}, y_{m}, \hat{\bar{u}}_{m, g}$ on $t$ is omitted in the figure.

The modulator $\bar{f}_{k}$ at transmitter $k$ is a linear map, producing the channel input

$$
x_{k}[t] \triangleq \bar{f}_{k}\left(\left(\bar{w}_{k, g}[t]\right)_{g \in \mathcal{G}_{L}}\right) \triangleq B \sum_{g \in \mathcal{G}_{L}} \bar{w}_{k, g}[t] g
$$

with

$$
B=B(L, p) \triangleq(K p)^{\left|\mathcal{G}_{L+1}\right|}
$$

For $g \in \mathcal{G}_{L+1}$, define

$$
\bar{u}_{m, g}[t] \triangleq \sum_{k=1}^{K} \bar{w}_{k,\left(g / h_{m, k}\right)}[t],
$$

where we use the convention that $\bar{w}_{k,\left(g / h_{m, k}\right)}[t]=0$ whenever $g / h_{m, k} \notin \mathcal{G}_{L}$.

The definition of $\bar{u}_{m, g}[t]$ can be interpreted in the following way. Let $\tilde{g} \in \mathcal{G}_{L}$, and consider the term $\bar{w}_{k, \tilde{g}}[t] \tilde{g}$ in the definition of $x_{k}[t]$. At receiver $m$, this term is observed as $\bar{w}_{k, \tilde{g}}[t] \tilde{g} h_{m, k}$. Thus, for any $g \in \mathcal{G}_{L+1}, \bar{u}_{m, g}[t]$ is the sum of all input subsymbols $\left(\bar{w}_{k, \tilde{g}}[t]\right)_{\tilde{g} \in \mathcal{G}_{L}}$ that are observed with coefficient $g$ at receiver $m$. Another way to see this is as follows. The signal observed at receiver $m$ is

$$
y_{m}=B \sum_{k=1}^{K} \sum_{g \in \mathcal{G}_{L}} \bar{w}_{k, g}[t] h_{m, k} g+z_{m} \text {. }
$$


Now, note that $h_{m, k} g$ is a monomial in the channel gains with highest exponent at most $L$. Hence $h_{m, k} g \in$ $\mathcal{G}_{L+1}$ for all $m, k$. Using the definition of $\bar{u}_{m, g}$, we can rewrite the received signal as

$$
\begin{aligned}
y_{m} & =B \sum_{k=1}^{K} \sum_{g \in \mathcal{G}_{L+1}} \bar{w}_{k,\left(g / h_{m, k}\right)}[t] g+z_{m} \\
& =B \sum_{g \in \mathcal{G}_{L+1}} \bar{u}_{m, g}[t] g+z_{m} .
\end{aligned}
$$

This last equation is a key step in the construction of the achievable scheme. It shows that the received signal can be decomposed into $\left|\mathcal{G}_{L+1}\right|$ terms $\bar{u}_{m, g}$, each multiplied by a different effective channel gain $g$. Crucially, each of these terms is an integer linear combination of up to $K$ input signals $\bar{w}_{k,\left(g / h_{m, k}\right)}$, one from each transmitter.

The demodulator $\bar{\phi}_{m}$ at receiver $m$ is the maximum likelihood detector of $\left(\bar{u}_{m, g}[t]\right)_{g \in \mathcal{G}_{L+1}}$, i.e.,

$$
\bar{\phi}_{m}\left(y_{m}[t]\right) \triangleq \underset{\left(\hat{\bar{u}}_{m, g}\right)}{\arg \max } \mathbb{P}\left(\cap_{g \in \mathcal{G}_{L+1}}\left\{\bar{u}_{m, g}[t]=\hat{\bar{u}}_{m, g}\right\} \mid y_{m}[t]\right)
$$

where the arg max is over all possible values of $\left(\hat{\bar{u}}_{m, g}\right)_{g \in \mathcal{G}_{L+1}}$. Denote by

$$
\left(\hat{\bar{u}}_{m, g}[t]\right)_{g \in \mathcal{G}_{L+1}} \triangleq \bar{\phi}_{m}\left(y_{m}[t]\right)
$$

the output of the demodulator. The probability of demodulation error at receiver $m$ is then defined as

$$
\mathbb{P}\left(\cup_{g \in \mathcal{G}_{L+1}}\left\{\hat{\bar{u}}_{m, g}[t] \neq \bar{u}_{m, g}[t]\right\}\right) .
$$

Observe that the goal of the demodulator is to recover $\left|\mathcal{G}_{L+1}\right|$ integers $\left(\bar{u}_{m, g}[t]\right)_{g}$ from a single observation $y_{m}[t]$.

The next lemma describes the performance of this modulation scheme.

Lemma 5. For any $K \geq 2$ and almost every $\boldsymbol{H} \in \mathbb{R}^{K \times K}$ there exist positive constants $c_{4}=c_{4}(K, \boldsymbol{H})$ and $c_{5}=c_{5}(K, \boldsymbol{H})$ such that, for all $p, L, t \in \mathbb{N}$ and $k \in\{1, \ldots, K\}$, the input signal to the channel has power at most

$$
\begin{aligned}
x_{k}^{2}[t] & \leq c_{4}^{L}(K p)^{2\left|\mathcal{G}_{L+1}\right|} L^{2 K^{2}} p^{2} \\
& \triangleq P(L, p)
\end{aligned}
$$

and

$$
\bigcup_{g \in \mathcal{G}_{L+1}}\left\{\hat{\bar{u}}_{m, g}[t] \neq \bar{u}_{m, g}[t]\right\} \subset\left\{\left|z_{m}[t]\right|>c_{5} p\right\},
$$

implying that the probability of demodulation error is at most

$$
\begin{aligned}
\mathbb{P}\left(\cup_{g \in \mathcal{G}_{L+1}}\left\{\hat{\bar{u}}_{m, g}[t] \neq \bar{u}_{m, g}[t]\right\}\right) & \leq \mathbb{P}\left(\left\{\left|z_{m}[t]\right|>c_{5} p^{1 / 2}\right\}\right) \\
& \leq \exp \left(-\frac{1}{2} c_{5}^{2} p\right) \\
& \triangleq \varepsilon(p) .
\end{aligned}
$$

The proof of Lemma 5 is presented in Section VI-C.

Lemma 5 bounds the power of the channel input $x_{k}[t]$ and, more importantly, states that the probability of demodulating in error decreases exponentially in $p$. Thus, when $p$ is large enough, the probability of demodulation error is small.

The original channel between transmitters and receivers produces noisy real linear combinations of the channel inputs. After applying the modulation scheme, we have transformed this into a channel that 
produces noisy integer linear combinations of the channel inputs. More precisely, using the definition of $\bar{u}_{m, g}$ in (24), we can write this new channel as

$$
\begin{aligned}
\hat{\bar{u}}_{m, g}[t] & =\bar{u}_{m, g}[t]+\bar{z}_{m, g}[t] \\
& =\sum_{k=1}^{K} \bar{w}_{k,\left(g / h_{m, k}\right)}[t]+\bar{z}_{m, g}[t],
\end{aligned}
$$

where we have defined the modulation noise $\bar{z}_{m, g}[t]$ as

$$
\bar{z}_{m, g}[t] \triangleq \hat{\bar{u}}_{m, g}[t]-\bar{u}_{m, g}[t] .
$$

Thus, we see that the channel resulting between the input of the modulator and the output of the demodulator computes noisy linear combinations with integer (indeed, either zero or one) coefficients.

We refer to this new channel after modulation as the modulated channel. Since the modulation scheme operates on a single time slot $t$, this modulated channel is discrete and memoryless. Note that the noise $\bar{z}_{m, g}$ of this modulated channel is not necessarily additive, i.e., $\bar{z}_{m, g}[t]$ is not necessarily independent of the channel input $\bar{u}_{m, g}[t]$. However, by Lemma 5, we know that the noise is small. This modulated channel is depicted in Fig. 4

As we have argued before, the probability of demodulation error $\varepsilon(p)$ goes to zero as $p \rightarrow \infty$ and hence, by (25), as power $P \rightarrow \infty$. However, the definition of computation capacity requires that the probability of error be arbitrarily small for fixed power $P$. The next step is therefore to transform the modulated channel into a system producing noiseless integer linear combinations of the channel inputs. To this end, we employ an outer code over the modulated channel. We call the encoder and decoder of this channel code $f_{k}$ and $\phi_{m}$ for transmitter $k$ and receiver $m$, respectively. It will be convenient to choose $p$ to be a prime number. Reducing the modulator outputs modulo $p$, we can then interpret the input and output subsymbols $\bar{w}_{k, g}[t]$ and $\hat{\bar{u}}_{m, g}[t]$ as well as the integer linear combinations performed by the channel as being in the finite field $\mathbb{F}_{p}$. We refer to the resulting channel over $\mathbb{F}_{p}$ as the modulated $\mathbb{F}_{p}$ channel.

We now describe the operations of the outer code in more detail (see Fig. 5). The channel encoder $f_{k}$ at transmitter $k$ consists of $\left|\mathcal{G}_{L}\right|$ sub-encoders

$$
f_{k} \triangleq\left\{f_{k, g}\right\}_{g \in \mathcal{G}_{L}}
$$

The channel decoder $g_{m}$ at receiver $m$ consists of $\left|\mathcal{G}_{L+1}\right|$ sub-decoders

$$
\begin{aligned}
& \phi_{m} \triangleq\left\{\phi_{m, g}\right\}_{g \in \mathcal{G}_{L+1}} . \\
& \left(\boldsymbol{w}_{1, g}\right)_{g \in \mathcal{G}_{L}} \rightarrow f_{1} \rightrightarrows\left(\overline{\boldsymbol{w}}_{1, g}\right)_{g \in \mathcal{G}_{L}} \vec{\longrightarrow} \\
& \left(\boldsymbol{w}_{2, g}\right)_{g \in \mathcal{G}_{L}} \Rightarrow f_{2} \rightarrow\left(\overline{\boldsymbol{w}}_{2, g}\right)_{g \in \mathcal{G}_{L}} \rightarrow \begin{array}{c}
\text { Modulated } \\
\text { Channel }
\end{array}\left(\hat{\boldsymbol{u}}_{2, g}\right)_{g \in \mathcal{G}_{L+1}} \rightrightarrows \phi_{2} \rightarrow\left(\hat{\boldsymbol{u}}_{2, g}\right)_{g \in \mathcal{G}_{L+1}} \\
& \left(\boldsymbol{w}_{3, g}\right)_{g \in \mathcal{G}_{L}} \rightarrow f_{3} \rightarrow\left(\overline{\boldsymbol{w}}_{3, g}\right)_{g \in \mathcal{G}_{L}} \rightarrow \quad
\end{aligned}
$$

Fig. 5. Outer code $\left(\left\{f_{k}\right\},\left\{\phi_{m}\right\}\right)$ over the modulated $\mathbb{F}_{p}$ channel. Each encoder $f_{k}$ uses the same linear map given by the matrix $\boldsymbol{S} \in \mathbb{F}_{p}^{T \times T R / \log (p)} . \phi_{m}$ is the corresponding minimum distance decoder for the equations $\boldsymbol{u}_{m}$.

Consider the message $w_{k, g}$ at sub-encoder $g$ of transmitter $k$. It will be convenient in the following to express this message as a vector in $\mathbb{F}_{p}^{T R / \log (p)}$. Whenever this vector structure is relevant, we will write the message as $\boldsymbol{w}_{k, g}$. The encoder $f_{k, g}$ maps $\boldsymbol{w}_{k, g}$ to the vector (or, equivalently, sequence) of modulator inputs

$$
\overline{\boldsymbol{w}}_{k, g} \triangleq\left(\bar{w}_{k, g}[t]\right)_{t=1}^{T} \in \mathbb{F}_{p}^{T}
$$

The rate of this channel encoder is hence

$$
\frac{\log \left(p^{T R / \log (p)}\right)}{T}=R
$$


bits per use of the modulated channel. The encoder is specified by the linear map

$$
\overline{\boldsymbol{w}}_{k, g} \triangleq f_{k, g}\left(\boldsymbol{w}_{k, g}\right) \triangleq \boldsymbol{S} \boldsymbol{w}_{k, g},
$$

for some matrix $S \in \mathbb{F}_{p}^{T \times T R / \log (p)}$, and where the multiplication is understood to be over $\mathbb{F}_{p}$. We point out that $S$ does not depend on $k$ and $g$. In other words, each encoder $f_{k, g}$ uses the same linear map.

Define the vector version of the demodulator output $\hat{\bar{u}}_{m, g}$ as

$$
\hat{\overline{\boldsymbol{u}}}_{m, g} \triangleq\left(\hat{\bar{u}}_{m, g}[t]\right)_{t=1}^{T} \in \mathbb{F}_{p}^{T} .
$$

Similar to the definition of $\bar{u}_{m, g}[t]$ in (24), set

$$
\boldsymbol{u}_{m, g} \triangleq \sum_{k=1}^{K} \boldsymbol{w}_{k,\left(g / h_{m, k}\right)}(\bmod p),
$$

where we again use the convention that $\boldsymbol{w}_{m,\left(g / h_{m, k}\right)}=\mathbf{0}$ whenever $g / h_{m, k} \notin \mathcal{G}_{L}$.

Recall that the modulated channel computes noisy linear combinations over the finite field $\mathbb{F}_{p}$. Since all channel encoders use the same linear code, this implies that the output of the subchannel $g$ at receiver $m$ is equal to $\boldsymbol{S} \boldsymbol{u}_{m, g}$ plus small noise $\overline{\boldsymbol{z}}_{m, g}$ resulting from erroneous demodulation as defined in (28). As pointed out earlier, the noise term $\overline{\boldsymbol{z}}_{m, g}$ may not be additive, i.e., $\overline{\boldsymbol{z}}_{m, g}$ may be dependent on the channel inputs. Formally, the (vector) of demodulated equations $\hat{\overline{\boldsymbol{u}}}_{m, g}$ is equal to

$$
\begin{aligned}
& \hat{\overline{\boldsymbol{u}}}_{m, g} \stackrel{(a)}{=} \sum_{k=1}^{K} \overline{\boldsymbol{w}}_{k,\left(g / h_{m, k}\right)}+\overline{\boldsymbol{z}}_{m, g} \\
& \stackrel{(b)}{=} \sum_{k=1}^{K} \boldsymbol{S} \boldsymbol{w}_{k,\left(g / h_{m, k}\right)}+\overline{\boldsymbol{z}}_{m, g} \\
& =\boldsymbol{S} \sum_{k=1}^{K} \boldsymbol{w}_{k,\left(g / h_{m, k}\right)}+\overline{\boldsymbol{z}}_{m, g} \\
& \stackrel{(c)}{=} \boldsymbol{S} \boldsymbol{u}_{m, g}+\overline{\boldsymbol{z}}_{m, g} \quad(\bmod p),
\end{aligned}
$$

where (a) follows (27), (b) follows from the definition of the encoder $f_{k, g}$ in (29), and (c) follows from the definition of the equation $\boldsymbol{u}_{m, g}$ in (30). Thus, since all transmitters use the same linear code, the encoding operation commutes with the operation of the channel. Note that (24) and (31) imply that

$$
\overline{\boldsymbol{u}}_{m, g}=\boldsymbol{S} \boldsymbol{u}_{m, g} .
$$

The decoder $\phi_{m, g}$ of the outer code is the minimum (Hamming) distance decoder, i.e.,

$$
\phi_{m, g}\left(\hat{\overline{\boldsymbol{u}}}_{m, g}\right) \triangleq \underset{\hat{\boldsymbol{u}}_{m, g} \in \mathbb{F}_{p}^{T R / \log (p)}}{\arg \min } \sum_{t=1}^{T} \mathbb{1}\left(\hat{\bar{u}}_{m, g}[t] \neq\left(\boldsymbol{S} \hat{\boldsymbol{u}}_{m, g}\right)[t]\right),
$$

where $\left(\boldsymbol{S} \hat{\boldsymbol{u}}_{m, g}\right)[t]$ is component $t$ of the vector $\boldsymbol{S} \hat{\boldsymbol{u}}_{m, g}$. Note that this decoder might not be the same as the maximum likelihood decoder, depending on the distribution of $\overline{\boldsymbol{z}}_{m, g}$. Denote by

$$
\hat{\boldsymbol{u}}_{m, g} \triangleq \phi_{m, g}\left(\hat{\overline{\boldsymbol{u}}}_{m, g}\right)
$$

the output of the decoder of the outer code. The probability of error of this code is defined as

$$
\mathbb{P}\left(\cup_{m, g}\left\{\hat{\boldsymbol{u}}_{m, g} \neq \boldsymbol{u}_{m, g}\right\}\right) .
$$

For $x \in(0,1)$, define the $p$-ary entropy function $\mathcal{H}_{p}(x)$ as

$$
\mathcal{H}_{p}(x) \triangleq \frac{1}{\log (p)}(x \log (p-1)-x \log (x)-(1-x) \log (1-x)) .
$$


The next lemma states that for the modulated $\mathbb{F}_{p}$ channel there exist linear codes $S$ with large rate that allow reliable decoding at each receiver.

Lemma 6. Denote by $\varepsilon(p)$ the upper bound on the probability of demodulation error as defined in (26) in Lemma 5] For every prime number $p$ such that $\varepsilon(p)<1 / 4$, and every $\eta \in(0,1 / 2-2 \varepsilon(p))$ there exists a linear code $\boldsymbol{S}$ (of sufficiently large blocklength $T$ ) for the modulated $\mathbb{F}_{p}$ channel with rate bigger than

$$
\left(1-\mathcal{H}_{p}(2 \varepsilon(p)+\eta)\right) \log (p)
$$

and probability of error less than $\eta$.

The proof of Lemma 6 is presented in Section VI-D.

Lemma 6 shows that, asymptotically in the blocklength $T$, reliable communication over the modulated subchannels is possible at rates arbitrarily close to

$$
\left(1-\mathcal{H}_{p}(2 \varepsilon(p))\right) \log (p),
$$

with probability of demodulation error $\varepsilon(p)$ as defined in Lemma 5. Since there are $K$ transmitters each with $\left|\mathcal{G}_{L}\right|$ subchannels, the sum rate achieved with this coding scheme is at least

$$
K\left|\mathcal{G}_{L}\right|\left(1-\mathcal{H}_{p}(2 \varepsilon(p))\right) \log (p) .
$$

Note that

$$
\lim _{p \rightarrow \infty} \mathcal{H}_{p}(2 \varepsilon(p))=0
$$

so that the sum rate is of order

$$
K\left|\mathcal{G}_{L}\right|(1-o(1)) \log (p)
$$

as $p \rightarrow \infty$.

To satisfy the definition of computation capacity, we need to argue that the mapping from $\left(\boldsymbol{w}_{k, g}\right)$ to $\left(\boldsymbol{u}_{m, g}\right)$ defined in (30) is deterministic and invertible over its range. As the channel gains $\boldsymbol{H}$ are constant and known, the mapping is clearly deterministic. The next lemma shows that the mapping is also injective (and hence invertible over its range).

Lemma 7. Let $p$ be a prime number. For any $K \geq 2$ and almost every $\boldsymbol{H} \in \mathbb{R}^{K \times K}$, the mapping from

$$
\left(\boldsymbol{w}_{k, g}: k \in\{1, \ldots, K\}, g \in \mathcal{G}_{L}\right)
$$

to

$$
\left(\boldsymbol{u}_{m, g}: m \in\{1, \ldots, K\}, g \in \mathcal{G}_{L+1}\right)
$$

is injective over $\mathbb{F}_{p}$.

The proof of Lemma 7 is presented in Section VI-E

Together with Lemmas 5 and 6, Lemma 7 shows that for every prime number $p$, a computation rate

$$
C(\boldsymbol{H}, P(L, p)) \geq K\left|\mathcal{G}_{L}\right|\left(1-\mathcal{H}_{p}(2 \varepsilon(p))\right) \log (p)
$$

is achievable, with

$$
P(L, p)=c_{4}^{L}(K p)^{2\left|\mathcal{G}_{L+1}\right|} L^{2 K^{2}} p^{2}
$$

as defined in (25).

Fix a power $P$, and let $p$ and $\tilde{p}$ be two consecutive prime numbers such that

$$
P(L, p) \leq P \leq P(L, \tilde{p})
$$

By Bertrand's postulate (see, for example, [28, Theorem 5.7.1]), any two consecutive primes $p$ and $\tilde{p}$ satisfy,

$$
p<\tilde{p} \leq 2 p .
$$


Since $P(L, p)$ is increasing in $p$, this implies that

$$
P(L, p) \leq P \leq P(L, 2 p) .
$$

Combining (34) and (35) shows that, for every power $P$ and corresponding prime number $p$ chosen as above,

$$
\frac{C(\boldsymbol{H}, P)}{\frac{1}{2} \log (P)} \geq \frac{C(\boldsymbol{H}, P(L, p))}{\frac{1}{2} \log (P(L, 2 p))}=D(p),
$$

where

$$
D(p) \triangleq \frac{K\left|\mathcal{G}_{L}\right|\left(1-\mathcal{H}_{p}(2 \varepsilon(p))\right) \log (p)}{\frac{1}{2} L \log \left(c_{4}\right)+\left|\mathcal{G}_{L+1}\right| \log (2 K p)+K^{2} \log (L)+\log (2 p)} .
$$

Since $p \rightarrow \infty$ as $P \rightarrow \infty$ (with $K$ and $L$ fixed), this implies that

$$
\liminf _{P \rightarrow \infty} \frac{C(\boldsymbol{H}, P)}{\frac{1}{2} \log (P)} \geq \lim _{p \rightarrow \infty} D(p)=\frac{K\left|\mathcal{G}_{L}\right|}{\left|\mathcal{G}_{L+1}\right|+1},
$$

where the limit $p \rightarrow \infty$ is understood as being taken over the prime numbers. By Remark 3 in Section IV] we have

$$
\left|\mathcal{G}_{L}\right|=L^{K^{2}},
$$

for almost every $\boldsymbol{H}$. Hence, this shows that

$$
\liminf _{P \rightarrow \infty} \frac{C(\boldsymbol{H}, P)}{\frac{1}{2} \log (P)} \geq \frac{K L^{K^{2}}}{(L+1)^{K^{2}+1}} .
$$

As this is true for all values of $L$, we may take the limit $L \rightarrow \infty$ to obtain

$$
\liminf _{P \rightarrow \infty} \frac{C(\boldsymbol{H}, P)}{\frac{1}{2} \log (P)} \geq \lim _{L \rightarrow \infty} \frac{K L^{K^{2}}}{(L+1)^{K^{2}}+1}=K .
$$

Hence, the proposed implementation of compute-and-forward achieves $K$ degrees of freedom, as needed to be shown.

We now derive an estimate of the rate of convergence to this limiting value. Fix a power $\tilde{P}$. Set

$$
L(\tilde{P}) \triangleq \log \frac{1}{1+K^{2}}(\tilde{P}),
$$

and let $p(\tilde{P})$ be the largest prime number $p$ such that $P(L(\tilde{P}), p) \leq \tilde{P}$. Using Bertrand's postulate as before, we obtain that

$$
P(L(\tilde{P}), p(\tilde{P})) \leq \tilde{P} \leq P(L(\tilde{P}), 2 p(\tilde{P})) .
$$

Solving $P(L, p)$ in (25) for $p$ yields

$$
p=\left(\frac{P}{c_{4}^{L} K^{2\left|\mathcal{G}_{L+1}\right|} L^{2 K^{2}}}\right)^{\frac{1}{2}\left(\left|\mathcal{G}_{L+1}\right|+1\right)^{-1}} .
$$

Together with (36), this implies that

$$
\log (p(\tilde{P}))=\frac{\log (\tilde{P})}{2\left(\left|\mathcal{G}_{L(\tilde{P})+1}\right|+1\right)}-\Theta(1),
$$

where we have used that $\left|\mathcal{G}_{L+1}\right|=(L+1)^{K^{2}}$.

${ }^{6}$ The number $L(\tilde{P})$ might not be an integer. We ignore the rounding error since it is immaterial as $\tilde{P} \rightarrow \infty$. 
From (34) and (36)

$$
\begin{aligned}
C(\boldsymbol{H}, \tilde{P}) & \geq C(\boldsymbol{H}, P(L(\tilde{P}), p(\tilde{P}))) \\
& =K\left|\mathcal{G}_{L}\right|\left(1-\mathcal{H}_{p}(2 \varepsilon(p))\right) \log (p),
\end{aligned}
$$

where we have suppressed dependence of $p$ and $L$ on $\tilde{P}$. By the definition of the $p$-ary entropy function $\mathcal{H}_{p}(x)$ in (33), we have for any $x \in(0,1)$

$$
\mathcal{H}_{p}(x) \leq x+\mathcal{H}_{2}(x) / \log (p) .
$$

Therefore, (38) can be further lower bounded as

$$
C(\boldsymbol{H}, \tilde{P}) \geq K\left|\mathcal{G}_{L}\right|\left((1-2 \varepsilon(p)) \log (p)-\mathcal{H}_{2}(2 \varepsilon(p))\right) .
$$

Substituting [37,

$$
C(\boldsymbol{H}, \tilde{P}) \geq \frac{K}{2}(1-2 \varepsilon(p)) \frac{\left|\mathcal{G}_{L}\right|}{\left|\mathcal{G}_{L+1}\right|+1} \log (\tilde{P})-\left|\mathcal{G}_{L}\right| \Theta(1) .
$$

From Lemma 5 ,

$$
1-2 \varepsilon(p(\tilde{P})) \geq 1-O\left(\log ^{-\frac{1}{1+K^{2}}}(\tilde{P})\right) .
$$

Moreover,

$$
\begin{aligned}
\frac{\left|\mathcal{G}_{L(\tilde{P})}\right|}{\left|\mathcal{G}_{L(\tilde{P})+1}\right|+1} & =\left(\left(1+\frac{1}{L(\tilde{P})}\right)^{K^{2}}+\frac{1}{(L(\tilde{P}))^{K^{2}}}\right)^{-1} \\
& \geq 1-O\left(\log ^{-\frac{1}{1+K^{2}}}(\tilde{P})\right)
\end{aligned}
$$

and

$$
\left|\mathcal{G}_{L(\tilde{P})}\right|=\log \frac{K^{2}}{1+K^{2}}(\tilde{P}) .
$$

Combining this with (39) yields

$$
\begin{aligned}
C(\boldsymbol{H}, \tilde{P}) & \geq \frac{K}{2}\left(1-O\left(\log ^{-\frac{1}{1+K^{2}}}(\tilde{P})\right)\right) \log (\tilde{P})-O\left(\log \frac{K^{2}}{1+K^{2}}(\tilde{P})\right) \\
& \geq \frac{K}{2} \log (\tilde{P})-O\left(\log \frac{K^{2}}{1+K^{2}}(\tilde{P})\right) .
\end{aligned}
$$

Thus, for every $K \geq 2$ and almost every $\boldsymbol{H} \in \mathbb{R}^{K \times K}$ there exists a positive constant $c_{2}=c_{2}(K, \boldsymbol{H})$ such that for all $\tilde{P} \geq 2$

$$
C(\boldsymbol{H}, \tilde{P}) \geq \frac{K}{2} \log (\tilde{P})-c_{2} \log \frac{K^{2}}{1+K^{2}}(\tilde{P}),
$$

completing the proof of the theorem.

\section{Proof of Lemma 5}

Since modulation involves only a mapping of one symbol at a time, we can drop the time indices in the following discussion (e.g., we write $x_{k}$ for $x_{k}[t]$ ).

We start by analyzing the power of the transmitted signal $x_{k}$. Each $\bar{w}_{k, g}$ takes value in $\{0, \ldots, p-1\}$ and hence has power $\bar{w}_{k, g}^{2} \leq p^{2}$. Moreover, $\left|\mathcal{G}_{L}\right|=L^{K^{2}}$, and each $g \in \mathcal{G}_{L}$ satisfies

$$
|g| \leq\left(\max \left\{1, \max _{\tilde{m}, \tilde{k}}\left|h_{\tilde{m}, \tilde{k}}\right|\right\}\right)^{L K^{2}} .
$$


Thus, the channel input $x_{k}$ as defined in (22) has power at most

$$
\begin{aligned}
x_{k}^{2} & =\left(B \sum_{g \in \mathcal{G}_{L}} \bar{w}_{k, g} g\right)^{2} \\
& \leq B^{2} L^{2 K^{2}} p^{2}\left(\max \left\{1, \max _{\tilde{m}, \tilde{k}}\left|h_{\tilde{m}, \tilde{k}}\right|\right\}\right)^{2 L K^{2}} .
\end{aligned}
$$

Defining the positive constant

$$
c_{4} \triangleq c_{4}(K, \boldsymbol{H}) \triangleq\left(\max \left\{1, \max _{\tilde{m}, \tilde{k}}\left|h_{\tilde{m}, \tilde{k}}\right|\right\}\right)^{2 K^{2}},
$$

and using the definition of $B$ in $(23)$, we obtain

$$
x_{k}^{2} \leq c_{4}^{L}(K p)^{2\left|\mathcal{G}_{L+1}\right|} L^{2 K^{2}} p^{2}
$$

as required.

We continue by analyzing the probability of demodulation error. Recall that the received signal

$$
\begin{aligned}
y_{m} & =\sum_{k=1}^{K} h_{m, k} x_{k}+z_{m} \\
& =B \sum_{k=1}^{K} \sum_{g \in \mathcal{G}_{L}} \bar{w}_{k, g} h_{m, k} g+z_{m}
\end{aligned}
$$

can be rewritten as

$$
y_{m}=B \sum_{g \in \mathcal{G}_{L+1}} \bar{u}_{m, g} g+z_{m},
$$

with $\bar{u}_{m, g}$ as defined in (24). Receiver $m$ aims to demodulate the functions $\left(\bar{u}_{m, g}\right)_{g \in \mathcal{G}_{L+1}}$ from $y_{m}$. We now argue that this is possible with small probability of error.

Consider a different set of linear combinations $\left(\bar{u}_{m, g}^{\prime}\right)_{g \in \mathcal{G}_{L+1}} \neq\left(\bar{u}_{m, g}\right)_{g \in \mathcal{G}_{L+1}}$, and compute the difference

$$
\left|\sum_{g \in \mathcal{G}_{L+1}}\left(\bar{u}_{m, g}-\bar{u}_{m, g}^{\prime}\right) g\right| \triangleq\left|\sum_{g \in \mathcal{G}_{L+1}} q_{g} g\right| .
$$

Note that $\bar{u}_{m, g} \in\{0, \ldots, K(p-1)\}$ and hence $q_{g} \in\{-K(p-1), \ldots, K(p-1)\}$. Moreover, observe that $\bar{u}_{m, g}=\bar{u}_{m, g}^{\prime}=0$ for $g=1$ in any valid set of equations and almost every $\boldsymbol{H}$, so that $q_{1}=0$ and can be ignored. By Lemma 4 for almost every $\boldsymbol{H}$ there is a finite constant $c=c(K, \boldsymbol{H})$ such that

$$
\left|\sum_{g \in \mathcal{G}_{L+1}, g \neq 1} q_{g} g\right| \geq c(K p)^{-\left|\mathcal{G}_{L+1}\right|+1 / 2}
$$

for all $\left(q_{g}\right)_{g \neq 1} \in\{-K(p-1), \ldots, K(p-1)\}^{\left|\mathcal{G}_{L+1}\right|-1},\left(q_{g}\right)_{g \neq 1} \neq \mathbf{0}$.

Combined with (40) and (41), this shows that the minimum distance between any two signal points at receiver $m$ is at least

$$
c B(K p)^{-\left|\mathcal{G}_{L+1}\right|+1 / 2} .
$$

Using the definition of $B$ in (23), we see that the minimum distance between the desired set of equations $\left(\bar{u}_{m, g}\right)_{g}$ and any other set of equations $\left(\bar{u}_{m, g}^{\prime}\right)_{g}$ is at least $c(K p)^{1 / 2}$. Therefore, the probability of demodulation error is upper bounded by

$$
\mathbb{P}\left(\cup_{g}\left\{\hat{\bar{u}}_{m, g} \neq \bar{u}_{m, g}\right\}\right) \leq \mathbb{P}\left(\left|z_{m}\right|>c_{5} p^{1 / 2}\right)
$$

with

$$
c_{5}=c_{5}(K, \boldsymbol{H}) \triangleq \frac{c K^{1 / 2}}{2} .
$$

This, in turn, can be upper bounded using the Chernoff bound as

$$
\mathbb{P}\left(\left|z_{1}\right|>c_{5} p^{1 / 2}\right) \leq \exp \left(-\frac{1}{2} c_{5}^{2} p\right),
$$

concluding the proof of the lemma. 


\section{Proof of Lemma 6}

We derive a lower bound on the rate achievable with linear codes over the modulated $\mathbb{F}_{p}$ channel. Recall that each channel encoder uses the same linear map $S$. By (31), the output of the sub-demodulator $g$ at receiver $m$ reduced modulo $p$ is

$$
\hat{\overline{\boldsymbol{u}}}_{m, g}=\boldsymbol{S} \boldsymbol{u}_{m, g}+\overline{\boldsymbol{z}}_{m, g} \quad(\bmod p),
$$

with (non-additive) noise $\overline{\boldsymbol{z}}_{m, g}$ satisfying

$$
\mathbb{1}\left(\bar{z}_{m, g}[t] \neq 0\right) \leq \mathbb{1}\left(\left|z_{m}[t]\right|>c_{5} p^{1 / 2}\right)
$$

for all $g \in \mathcal{G}_{L+1}$, and

$$
\mathbb{P}\left(\left|z_{m}[t]\right|>c_{5} p^{1 / 2}\right) \leq \varepsilon(p)=\varepsilon
$$

by Lemma 5. Thus, we only need to analyze the performance of linear codes over a point-to-point channel with input and output alphabets $\mathbb{F}_{p}$ and (non-additive) noise $\bar{z}_{m, g}[t]$.

By the Gilbert-Varshamov bound (see, e.g., [29, Theorem 12.3.2, Theorem 12.3.4]), for every $T$, prime number $p$, and $2 \leq d \leq T / 2$, there exists a linear block code of length $T$ over $\mathbb{F}_{p}$ with rate at least

$$
\left(1-\mathcal{H}_{p}((d-1) / T)\right) \log (p),
$$

and minimum distance at least $d$. Recall that $0 \leq \varepsilon<1 / 4$ by assumption, and fix $\eta \in(0,1 / 2-2 \varepsilon)$. Choose

$$
d=\lfloor(2 \varepsilon+\eta) T\rfloor \geq(2 \varepsilon+\eta) T-1,
$$

so that the linear code can correct up to

$$
\lfloor(d-1) / 2\rfloor \geq\lfloor(\varepsilon+\eta / 2) T-1\rfloor \geq(\varepsilon+\eta / 2) T-2
$$

errors.

Since we use minimum-distance decoding at the receivers, this implies that we make an error only if the noise has Hamming weight larger than $(\varepsilon+\eta / 2) T-2$, i.e., if

$$
\max _{g} \sum_{t=1}^{T} \mathbb{1}\left(\bar{z}_{m, g}[t] \neq 0\right)>(\varepsilon+\eta / 2) T-2 .
$$

Using (42), we have

$$
\mathbb{P}\left(\max _{g} \sum_{t=1}^{T} \mathbb{1}\left(\bar{z}_{m, g}[t] \neq 0\right)>(\varepsilon+\eta / 2) T-2\right) \leq \mathbb{P}\left(\sum_{t=1}^{T} \mathbb{1}\left(\left|z_{m}[t]\right| \geq c_{5} p^{1 / 2}\right)>(\varepsilon+\eta / 2) T-2\right) .
$$

Since $\left(z_{m}[t]\right)_{t}$ is i.i.d., the weak law of large numbers shows together with (43) that

$$
\lim _{T \rightarrow \infty} \mathbb{P}\left(\max _{g} \sum_{t=1}^{T} \mathbb{1}\left(\bar{z}_{m, g}[t] \neq 0\right)>(\varepsilon+\eta / 2) T-2\right)=0 .
$$

In particular, for $T$ large enough this probability is less than $\eta / K$, so that with probability at least $1-\eta$ all decoders are able to decode correctly. The rate of this code is at least

$$
\left(1-\mathcal{H}_{p}((d-1) / T)\right) \log (p) \geq\left(1-\mathcal{H}_{p}(2 \varepsilon+\eta)\right) \log (p),
$$

where we have used that $\mathcal{H}_{p}(x)$ is increasing in $x$ for $x \leq 1 / 2$. This proves the lemma.

\footnotetext{
${ }^{7}$ We point out that the law of large numbers does not apply to $\bar{z}_{m, g}[t]$, since this sequence is dependent on the channel input and therefore, without further assumptions on those inputs, not i.i.d.
} 


\section{E. Proof of Lemma 7}

We need to show that the map from the input to the encoder of the outer code

$$
\left(\boldsymbol{w}_{k, g}: k \in\{1, \ldots, K\}, g \in \mathcal{G}_{L}\right)
$$

to the correct output of the decoder of the outer code

$$
\left(\boldsymbol{u}_{m, \tilde{g}}: m \in\{1, \ldots, K\}, \tilde{g} \in \mathcal{G}_{L+1}\right)
$$

is injective. The mapping from $\boldsymbol{w}_{k, g}$ to $\overline{\boldsymbol{w}}_{k, g}$ defined in (29) and the mapping from $\boldsymbol{u}_{m, \tilde{g}}$ to $\overline{\boldsymbol{u}}_{m, \tilde{g}}$ given by (32) are both invertible over their range. Hence, it suffices to prove injectivity of the map from the input to the modulators

$$
\left(\overline{\boldsymbol{w}}_{k, g}: k \in\{1, \ldots, K\}, g \in \mathcal{G}_{L}\right)
$$

to the correct output of the demodulators

$$
\left(\overline{\boldsymbol{u}}_{m, \tilde{g}}: m \in\{1, \ldots, K\}, \tilde{g} \in \mathcal{G}_{L+1}\right)
$$

over $\mathbb{F}_{p}$. Finally, since this map is defined at the symbol level, it suffices to prove injectivity for a single time slot $t$. To simplify notation, we drop the index $t$ in the following, i.e., we write $\bar{w}_{k, g}$ and $\bar{u}_{m, \tilde{g}}$ for $\bar{w}_{k, g}[t]$ and $\bar{u}_{m, \tilde{g}}[t]$. Thus, we need to show that the map from

$$
\left(\bar{w}_{k, g}: k \in\{1, \ldots, K\}, g \in \mathcal{G}_{L}\right)
$$

to

$$
\left(\bar{u}_{m, \tilde{g}}: m \in\{1, \ldots, K\}, \tilde{g} \in \mathcal{G}_{L+1}\right)
$$

is invertible over its range. This map is defined in (24) as

$$
\bar{u}_{m, \tilde{g}}[t]=\sum_{k=1}^{K} \bar{w}_{k,\left(\tilde{g} / h_{m, k}\right)}[t] \quad(\bmod p),
$$

with the convention that $\bar{w}_{k, g}=0$ whenever $\bar{w}_{k, g} \notin \mathcal{G}_{L}$, and taking into account that the modulator output is reduced modulo $p$.

In the remainder of the proof, we make repeated use of the fact that, for almost every channel realization $\boldsymbol{H}$, every monomial (evaluated at $\boldsymbol{H}$ ) in $\mathcal{G}_{L+1}$ can be uniquely factorized into powers of $\left(h_{m, k}\right)$, see Remark 3 in Section IV] We refer to this as the unique factorization property in what follows. For $g \in \mathcal{G}_{L+1}$, we use the notation $h_{m, k}^{s} \mid g$ to denote that $h_{m, k}^{s}$ is a factor of $g$ in this unique factorization.

We begin with a small example with $L=K=2$. Recall that the channel gain between transmitter $k$ and receiver $m$ is denoted by $h_{m, k}$. By definition,

$$
\mathcal{G}_{2}=\left\{h_{1,1}^{s_{1,1}} h_{1,2}^{s_{1,2}} h_{2,1}^{s_{2,1}} h_{2,2}^{s_{2,2}}: s_{1,1}, s_{1,2}, s_{2,1}, s_{2,2} \in\{0,1\}\right\}
$$

and $\left|\mathcal{G}_{2}\right|=16$ for almost every $\boldsymbol{H}$ by unique factorization.

Consider the received monomial $\tilde{g} \in \mathcal{G}_{3}$. If

$$
h_{1,1}^{2} \mid \tilde{g}
$$

at receiver 1 , then $\tilde{g}$ can have originated only from transmitter 1 by unique factorization. In other words, $\bar{u}_{1, \tilde{g}}=\bar{w}_{1,\left(\tilde{g} / h_{1,1}\right)}$ and hence $\bar{w}_{1,\left(\tilde{g} / h_{1,1}\right)}$ can be recovered from the received signal. If

$$
h_{2,1}^{2} \mid \tilde{g}
$$

at receiver 2 than again $\tilde{g}$ can have originated only from transmitter 1 by unique factorization. Hence $\bar{u}_{2, \tilde{g}}=\bar{w}_{1,\left(\tilde{g} / h_{2,1}\right)}$ and $\bar{w}_{\left.1, \tilde{g} / h_{2,1}\right)}$ can be recovered. We can then remove these messages from all other equations at the receivers. 
A similar conclusion can be drawn if $h_{1,2}^{2}$ is a factor at receiver 1 or respectively $h_{2,2}^{2}$ a factor at receiver 2. In either case the monomial originated at transmitter 2 , and the corresponding message $\bar{w}_{2, g}$ can be recovered again by unique factorization and the terms again be removed from all received signals.

Other parts of the signals may also be identified as only originating from transmitter 1. For example $h_{1,1} h_{2,1}$ at receiver 1 cannot be seen as a message from transmitter 2 because $h_{1,2}$ is not a factor. All such messages can be decoded and removed as was done with the previous messages, again by unique factorization.

This leaves only the message $\bar{w}_{1, g}$ with $g=h_{1,2} h_{2,2}$ from transmitter 1 and the message $\bar{w}_{2, g}$ with $g=h_{1,1} h_{2,1}$ from transmitter 2 to be determined. But $h_{1,2} h_{2,2}$ from transmitter 1 is observed at receiver 1 as

$$
\tilde{g}=g h_{1,1}=h_{1,1} h_{1,2} h_{2,2}
$$

which, to have originated from transmitter 2, would be transmitted as

$$
\tilde{g} / h_{1,2}=h_{1,1} h_{2,2} \text {. }
$$

However, this message was already removed in the first round (since it is observed at receiver 2 as $h_{1,1} h_{2,2}^{2}$ ) and so the remaining signal at receiver 1 must have originated from transmitter 1 . Thus $\bar{w}_{1, g}$ can be determined. The same can be done for $g=h_{1,1} h_{2,1}$ from transmitter 2 , and the message $\bar{w}_{2, g}$ can be obtained. This completes the example as all messages $\bar{w}_{k, g}$ for $g \in \mathcal{G}_{2}, k \in\{1,2\}$ have been determined.

We now extend the above argument to $K=2$ but $L$ arbitrary, proceeding by induction. We will argue that the messages $\bar{w}_{k, g}, k=\{1,2\}, g \in \mathcal{G}_{L}$ are completely determined by $\bar{u}_{m, \tilde{g}}$ where $m \in\{1,2\}$ and $\tilde{g}$ ranges over the possible received monomials in $\mathcal{G}_{L+1}$. The proof is by induction on $L$, and our earlier argument for $L=2$ anchors the induction.

Suppose the induction hypothesis holds for $L-1 \geq 2$. We now show it holds for $L$ as well. As before, determine and remove from the received signals all messages $\bar{w}_{1, g}$, such that $h_{1,1}^{L-1} \mid g$ or $h_{2,1}^{L-1} \mid g$ at transmitter 1 (using unique factorization). Do the same, but for messages $\bar{w}_{2, g}$ such that $h_{1,2}^{L-1} \mid g$ or $h_{2,2}^{L-1} \mid g$ at transmitter 2 .

Now, consider $g$ at transmitter 1 such that $h_{1,2}^{L-1} \mid g$. This will be received as

$$
\tilde{g}=g h_{2,1}
$$

at receiver 2, and either $h_{2,2} \mid g$ or it is seen as a monomial component originating from transmitter 1 immediately by unique factorization. To be from transmitter 2 , the transmit monomial would be

$$
\tilde{g} / h_{2,2} \text {. }
$$

But then $h_{1,2}^{L-1} \mid\left(\tilde{g} / h_{2,2}\right)$ and therefore the message corresponding to this signal has already been removed from both receivers.

The same is true for all messages $\bar{w}_{1, g}$ with $g$ such that $h_{2,2}^{L-1} \mid g$ at transmitter 1 . Moreover the same arguments apply to transmitter 2 but with $h_{1,1}^{L-1}$ and $h_{2,1}^{L-1}$. It follows that all factors $\bar{w}_{k, g}$ with the highest exponent in $g$ being $L-1$ have been determined for both transmitters. The remaining monomials make up $\mathcal{G}_{L-1}$ at both transmitters. Since the factors involving monomials with highest exponent $L-1$ have been removed, we may apply the induction hypothesis to complete the inversion. Thus, for $K=2$ and arbitrary $L \geq 2$, the mapping can be inverted.

It remains to consider $K \geq 2$ and $L \geq 2$. We will argue that the factors $\bar{w}_{k, g}, k \in\{1,2, \cdots, K\}, g \in \mathcal{G}_{L}$ are completely determined by $\bar{u}_{m, \tilde{g}}$ where $m=\{1,2, \cdots, K\}$ and $\tilde{g}$ ranges over the possible received monomials in $\mathcal{G}_{L+1}$. As earlier, we proceed by induction, but this time on $K$. The result holds for $K=2$ as we have already demonstrated.

Suppose then the result holds for $K-1 \geq 2$, and consider the case with $K$ transmitters and receivers. Fix $L \geq 2$ arbitrarily. For each transmitter $k$, we can once again remove all the factors $\bar{w}_{k, g}$ whenever

$$
h_{m, k}^{L-1} \mid g
$$


for some $m$.

Now let us fix a receiver, say $\tilde{m}=1$, and a transmitter, say $\tilde{k}=2$. Note this choice is entirely arbitrary as transmitters are in no sense tied to receivers so we may re-index them to obtain this case. Consider a monomial $g$ at transmitter $k$ such that

$$
h_{1,2}^{L-1} \mid g .
$$

At receiver 2, this is observed as

$$
\tilde{g}=g h_{2, k} .
$$

For such a monomial to be seen at receiver 2 as originating from transmitter 2 , we must have that

$$
h_{2,2} \mid \tilde{g},
$$

since otherwise we can rule out transmitter 2 at receiver 2 by unique factorization. However, if this is the case, we see that the corresponding message has already been removed for transmitter 2 as

$$
h_{1,2}^{L-1} \mid\left(\tilde{g} / h_{2,2}\right) .
$$

Thus under either outcome this monomial component cannot be seen as originating from transmitter 2.

Proceed as follows. Consider receivers $m \in\{2,3, \cdots, K\}$ (leaving out receiver $\tilde{m}=1$ ) and collect all equations $\bar{u}_{m, \tilde{g}}$ such that $m \neq 1$ and

$$
h_{1,2}^{L-1} \mid \tilde{g}
$$

using unique factorization. We may consider these as originating only from transmitters $k \in\{1,3, \cdots, K\}$ (leaving out transmitter $\tilde{k}=2$ ) by the argument in the preceding paragraph. We thus now have the problem of identifying $\bar{w}_{k, g}$ such that $h_{1,2}^{L-1} \mid g, k \neq 1$, using the received signals at $m \neq 1$. Denote the corresponding set of transmit monomials by

$$
\mathcal{G}_{L}^{1,2} \triangleq\left\{g \in \mathcal{G}_{L}: h_{1,2}^{L-1} \mid g\right\} .
$$

Observe that any power of the channel gains $h_{m, 2}, m \neq 1$ and $h_{1, k}, k \neq 2$ may be a factor of the monomials in $\mathcal{G}_{L}^{1,2}$.

To proceed further, note that the monomials in $\mathcal{G}_{L}^{1,2}$ can be partitioned into equivalence classes such that each $g$ in the same class has the same factors

$$
h_{1,2}^{L-1} \prod_{k \neq 2} h_{1, k}^{s_{1, k}} \prod_{m \neq 1} h_{m, 2}^{s_{m, 2}}
$$

in their unique factorization for some fixed $0 \leq s_{1, k} \leq L-1,0 \leq s_{m, 2} \leq L-1$. We call (44) the $(1,2)$-factor of $g$. We may also partition the receive monomials according to their $(1,2)$-factor. Denote by

$$
\mathcal{G}_{L}^{1,2}\left(\left(s_{1, k}\right)_{k \neq 2},\left(s_{m, 2}\right)_{m \neq 1}\right) \subset \mathcal{G}_{L}^{1,2}
$$

the equivalence class with $(1,2)$-factor

$$
h_{1,2}^{L-1} \prod_{k \neq 2} h_{1, k}^{s_{1, k}} \prod_{m \neq 1} h_{m, 2}^{s_{m, 2}}
$$

Fix a class $\left(s_{1, k}\right)_{k \neq 2},\left(s_{m, 2}\right)_{m \neq 1}$, and consider the messages

$$
\left(\bar{w}_{k, g}: k \neq 2, g \in \mathcal{G}_{L}^{1,2}\left(\left(s_{1, k}\right)_{k \neq 2},\left(s_{m, 2}\right)_{m \neq 1}\right)\right)
$$

and the equations

$$
\left(\bar{u}_{m, \tilde{g}}: m \neq 1, \tilde{g} \in \mathcal{G}_{L+1}^{1,2}\left(\left(s_{1, k}\right)_{k \neq 2},\left(s_{m, 2}\right)_{m \neq 1}\right)\right) .
$$

Recall that we have removed all messages $\bar{w}_{2, g}$ from the equations (46). Observe that any equation $\bar{u}_{m, \tilde{g}}$ in (46) is then solely a function of the messages in (45). 
Divide out the common (1,2)-factor from the transmit and receive monomials in (45) and (46). This results in a set of messages and equations with monomials in the channel coefficients $h_{m, k}, k \neq 2, m \neq 1$ with $K-1$ transmitters and $K-1$ receivers. By our induction hypothesis, we may invert to obtain all $\bar{w}_{k, g}, g \in \mathcal{G}_{L}^{1,2}$, working with each $(1,2)$-factor class in turn.

However, the choice of $\tilde{k}=1, \tilde{m}=2$ plays no special role in the above argument as we have already explained, so that we may recover $\bar{w}_{k, g}$ for all monomials

$$
\left\{g \in \mathcal{G}_{L}: h_{\tilde{m}, \tilde{k}}^{L-1} \mid g\right\}
$$

and any choice $\tilde{k}, \tilde{m} \in\{1,2, \cdots, K\}$. Removing these decoded messages from the received equations, we have reduced the monomials to have exponent no higher than $L-2$. Hence, we may proceed iteratively, reducing the order of $L$ by one in each iteration. Thus, $\bar{w}_{k, g}$ can be recovered for all $k \in\{1,2, \cdots, K\}, g \in$ $\mathcal{G}_{L}$, that is, the mapping is invertible over its range.

\section{CONCLUSION}

We considered the asymptotic behavior of compute-and-forward over a section of a relay network with $K$ transmitters and $K$ relays. We showed that the lattice implementation of compute-and-forward proposed by Nazer and Gastpar in [11] achieves at most $2 /(1+1 / K) \leq 2$ degrees of freedom. Thus, the asymptotic behavior of the lattice scheme is very different from the MIMO upper bound resulting from allowing full cooperation among transmitters and among relays and achieving $K$ degrees of freedom. We then argued that this gap is not fundamental to the compute-and-forward approach in general, but rather due to the lattice implementation in [11]. To this end, we proposed and analyzed a different implementation of compute-and-forward and showed that it achieves $K$ degrees of freedom. Thus, at least in terms of degrees of freedom, compute-and-forward can achieve the same asymptotic rates as if full cooperation among transmitters and among relays were permitted.

\section{ACKNOWLEDGMENTS}

The authors would like to thank the anonymous reviewers for their careful reading of the manuscript and their thoughtful comments.

\section{APPENDIX A}

\section{Change of Measure in the Proof of Theorem 1}

Here we show that if (21) in Section $\nabla$ holds for almost all $\tilde{\boldsymbol{h}} \in \mathbb{R}^{K-1}$, then it also holds for almost all $\boldsymbol{h} \in \mathbb{R}^{K}$. In the following discussion, we use the notation $\mu_{K}$ to denote Lebesgue measure over $\mathbb{R}^{K}$. Let $B \subset \mathbb{R}^{K-1}$ be a set of vectors $\tilde{\boldsymbol{h}} \in \mathbb{R}^{K-1}$ of measure zero, i.e.,

$$
\mu_{K-1}(B)=0 \text {. }
$$

Let $D \subset \mathbb{R}^{K}$ be the set of vectors $\boldsymbol{h} \in \mathbb{R}^{K}$ such that

$$
\frac{1}{\|\boldsymbol{h}\|}\left(h_{1} \quad \cdots \quad h_{K-1}\right) \in B .
$$

We want to show that $D$ has also measure zero, i.e., $\mu_{K}(D)=0$. We have

$$
\begin{aligned}
\mu_{K}(D) & =\int_{\boldsymbol{h} \in \mathbb{R}^{K}} \mathbb{1}_{D}(\boldsymbol{h}) d \boldsymbol{h} \\
& =\int_{\boldsymbol{h} \in \mathbb{R}^{K}} \mathbb{1}_{B}\left(\frac{1}{\|\boldsymbol{h}\|}\left(h_{1} \cdots \cdots h_{K-1}\right)\right) d \boldsymbol{h} .
\end{aligned}
$$


Making the change of variables

$$
\begin{aligned}
\tilde{h}_{k} & \triangleq \frac{h_{k}}{\|\boldsymbol{h}\|}, \quad \text { for } k \in\{1, \ldots, K-1\}, \\
s & \triangleq\|\boldsymbol{h}\|,
\end{aligned}
$$

and using the nonnegativity of $\mathbb{1}_{B}$ together with Fubini's theorem, we can rewrite this as

$$
\mu_{K}(D)=\int_{s=0}^{\infty} s^{K-1} \int_{\tilde{\boldsymbol{h}} \in \mathbb{R}^{K-1}:\|\tilde{\boldsymbol{h}}\| \leq 1} \mathbb{1}_{B}(\tilde{\boldsymbol{h}})\left(1-\|\tilde{\boldsymbol{h}}\|^{2}\right)^{-1 / 2} d \tilde{\boldsymbol{h}} d s .
$$

Now,

$$
\begin{aligned}
\int_{\tilde{\boldsymbol{h}} \in \mathbb{R}^{K-1}:\|\tilde{\boldsymbol{h}}\| \leq 1} & \mathbb{1}_{B}(\tilde{\boldsymbol{h}})\left(1-\|\tilde{\boldsymbol{h}}\|^{2}\right)^{-1 / 2} d \tilde{\boldsymbol{h}} \\
& \leq \int_{\tilde{\boldsymbol{h}} \in \mathbb{R}^{K-1}:\|\tilde{\boldsymbol{h}}\| \leq \sqrt{1-\varepsilon^{2}}} \mathbb{1}_{B}(\tilde{\boldsymbol{h}}) \varepsilon^{-1} d \tilde{\boldsymbol{h}}+\int_{\tilde{\boldsymbol{h}} \in \mathbb{R}^{K-1}: \sqrt{1-\varepsilon^{2}}<\|\tilde{\boldsymbol{h}}\| \leq 1}\left(1-\|\tilde{\boldsymbol{h}}\|^{2}\right)^{-1 / 2} d \tilde{\boldsymbol{h}} \\
& \leq \varepsilon^{-1} \mu_{K-1}(B)+2 \frac{\pi^{(K-1) / 2}}{\Gamma((K-1) / 2)} \int_{\sqrt{1-\varepsilon^{2}}<\tilde{s} \leq 1}\left(1-\tilde{s}^{2}\right)^{-1 / 2} d \tilde{s} \\
& =2 \frac{\pi^{(K-1) / 2}}{\Gamma((K-1) / 2)}\left(\pi / 2-\arcsin \left(\sqrt{1-\varepsilon^{2}}\right)\right)
\end{aligned}
$$

for every $\varepsilon>0$, and where $\Gamma(\cdot)$ denotes the Gamma function. Letting $\varepsilon \rightarrow 0$, we obtain

$$
\int_{\tilde{\boldsymbol{h}} \in \mathbb{R}^{K-1}:\|\tilde{\boldsymbol{h}}\| \leq 1} \mathbb{1}_{B}(\tilde{\boldsymbol{h}})\left(1-\|\tilde{\boldsymbol{h}}\|^{2}\right)^{-1 / 2} d \tilde{\boldsymbol{h}}=0,
$$

and hence

$$
\mu_{K}(D)=0 .
$$

This shows that (21) holds also for almost every $\boldsymbol{H} \in \mathbb{R}^{K \times K}$.

\section{REFERENCES}

[1] T. M. Cover and A. A. El Gamal, "Capacity theorems for the relay channel," IEEE Trans. Inf. Theory, vol. 25, pp. 572-584, Sept. 1972.

[2] J. N. Laneman, D. N. C. Tse, and G. W. Wornell, "Cooperative diversity in wireless networks: Efficient protocols and outage behaviour," IEEE Trans. Inf. Theory, vol. 50, pp. 3062-3080, Dec. 2004.

[3] G. Kramer, M. Gastpar, and P. Gupta, "Cooperative strategies and capacity theorems for relay networks," IEEE Trans. Inf. Theory, vol. 51, pp. 3037-3063, Sept. 2005.

[4] B. Schein and R. Gallager, "The Gaussian parallel relay network," in Proc. IEEE ISIT, p. 22, June 2000.

[5] M. Gastpar and M. Vetterli, "On the capacity of large Gaussian relay networks," IEEE Trans. Inf. Theory, vol. 51, pp. 765-779, Mar. 2005.

[6] U. Niesen and S. Diggavi, “The approximate capacity of the Gaussian N-relay diamond network," arXiv:1008.3813 [cs.IT], Aug. 2010. To appear in IEEE Trans. Inf. Theory.

[7] Y.-H. Kim, "Capacity of a class of deterministic relay channels," IEEE Trans. Inf. Theory, vol. 54, pp. 1328-1329, Mar. 2008.

[8] M. Aleksic, P. Razahgi, and W. Yu, "Capacity of a class of modulo-sum relay channels," IEEE Trans. Inf. Theory, vol. 55, pp. 921-930, Mar. 2009.

[9] A. Sanderovich, O. Somekh, H. V. Poor, and S. Shamai, "Uplink macro diversity of limited backhaul cellular network," IEEE Trans. Inf. Theory, vol. 55, pp. 3457-3478, Aug. 2009.

[10] A. S. Avestimehr, S. N. Diggavi, and D. N. C. Tse, "Wireless network information flow: A deterministic approach," IEEE Trans. Inf. Theory, vol. 57, pp. 1872-1905, Apr. 2011.

[11] B. Nazer and M. Gastpar, "Compute-and-forward: Harnessing interference through structured codes," IEEE Trans. Inf. Theory, vol. 57, pp. 6463-6484, Oct. 2011.

[12] W. Nam, S.-Y. Chung, and Y. H. Lee, "Capacity of the Gaussian two-way relay channel to within $1 / 2$ bit," IEEE Trans. Inf. Theory, vol. 56, pp. 5488-5494, Nov. 2010.

[13] S. Zhang, S. C. Liew, and P. P. Lam, "Hot topic: Physical-layer network coding," in Proc. ACM MobiCom, pp. 358-365, Sept. 2006.

[14] M. P. Wilson, K. Narayanan, H. D. Pfister, and A. Sprintson, "Joint physical layer coding and network coding for bidirectional relaying," IEEE Trans. Inf. Theory, vol. 56, pp. 5641-5654, Nov. 2010. 
[15] S. Katti, S. Gollakota, and D. Katabi, "Embracing wireless interference: Analog network coding," in Proc. ACM SIGCOMM, pp. 397408, Oct. 2007.

[16] H.-A. Loeliger, "Averaging bounds for lattices and linear codes," IEEE Trans. Inf. Theory, vol. 43, pp. 1767-1773, Nov. 1997.

[17] U. Erez and R. Zamir, "Achieving $\frac{1}{2} \log (1+\mathrm{SNR})$ on the AWGN channel with lattice encoding and decoding," IEEE Trans. Inf. Theory, vol. 50, pp. 2293-2314, Oct. 2004.

[18] M. A. Maddah-Ali, "On the degrees of freedom of the compound MISO broadcast channels with finite states," in Proc. IEEE ISIT, pp. 2273-2277, June 2010 .

[19] D. Kleinbock, "Baker-Sprindžuk conjectures for complex analytic manifolds," in Algebraic Groups and Arithmetic (S. G. Dani and G. Prasad, eds.), pp. 539-553, Narosa Publishing House, Apr. 2004.

[20] A. S. Motahari, S. O. Gharan, M. A. Maddah-Ali, and A. K. Khandani, "Real interference alignment: Exploiting the potential of single antenna systems," arXiv:0908.2282 [cs.IT], Aug. 2009. Submitted to IEEE Trans. Inf. Theory.

[21] A. Høst-Madsen and A. Nosratinia, "The multiplexing gain of wireless networks," in Proc. IEEE ISIT, pp. 2065-2069, Sept. 2005.

[22] I. E. Telatar, "Capacity of multi-antenna Gaussian channels," Europ. Trans. Telecommun., vol. 10, pp. 585-595, November 1999.

[23] R. H. Etkin and E. Ordentlich, "The degrees-of-freedom of the $K$-user Gaussian interference channel is discontinuous at rational channel coefficients," IEEE Trans. Inf. Theory, vol. 55, pp. 4932-4946, Nov. 2009.

[24] V. Bernik, D. Kleinbock, and G. A. Margulis, "Khintchine-type theorems on manifolds: The convergence case for standard and multiplicative versions," Int. Math. Res. Notices, vol. 2001, no. 9, pp. 453-486, 2001.

[25] V. Beresnevich, "A Groshev type theorem for convergence on manifolds," Acta Math. Hungar., vol. 94, pp. 99-130, May 2002.

[26] V. G. Sprindžuk, Metric Theory of Diophantine Approximations. Wiley, 1979.

[27] R. Durret, Probability: Theory and Examples. Duxbury Press, third ed., 2004.

[28] L.-K. Hua, Introduction to Number Theory. Springer, 1982.

[29] R. E. Blahut, Algebraic Codes for Data Transmission. Cambridge University Press, 2003. 